\title{
Modeling Swarm Robotic Systems: A Case Study in Collaborative Distributed Manipulation
}

\author{
Alcherio Martinoli ${ }^{1}$, Kjerstin Easton $^{2}$, and William Agassounon ${ }^{3}$ \\ ${ }^{1}$ Swarm-Intelligent Systems Group, Nonlinear Systems Laboratory, EPFL, 1015 Lausanne, Switzerland \\ ${ }^{2}$ Robotics Laboratory, California Institute of Technology, Pasadena, CA 91125, U.S.A. \\ ${ }^{3}$ Physical Sciences Inc., 20 New England Business Center, Andover, MA 01810, U.S.A. \\ E-mail: alcherio.martinoli@epfl.ch, easton@caltech.edu, agassounon@psicorp.com
}

\begin{abstract}
In this paper, we present a time-discrete, incremental methodology for modeling, at the microscopic and macroscopic level, the dynamics of distributed manipulation experiments using swarms of autonomous robots endowed with reactive controllers. The methodology is well-suited for nonspatial metrics since it does not take into account robots' trajectories or the spatial distribution of objects in the environment. The strength of the methodology lies in the fact that it has been generated by considering incremental abstraction steps, from real robots to macroscopic models, each with well-defined mappings between successive implementation levels. Precise heuristic criteria based on geometrical considerations and systematic tests with one or two real robots prevent the introduction of free parameters in the calibration procedure of models. As a consequence, we are able to generate highly abstracted macroscopic models that can capture the dynamics of a swarm of robots at the behavioral level while still being closely anchored to the characteristics of the physical set-up. Although this methodology has been and can be applied to other experiments in distributed manipulation (e.g., object aggregation and segregation, foraging), in this paper we focus on a strictly collaborative case study concerned with pulling sticks out of the ground, an action that requires the collaboration of two robots to be successful. Experiments were carried out with teams consisting of two to 600 individuals at different levels of implementation (real robots, embodied simulations, microscopic and macroscopic models). Results show that models can deliver both qualitatively and quantitatively correct predictions in time lapses that are at least four orders of magnitude smaller than those required by embodied simulations and that they represent a useful tool for generalizing the dynamics of these highly stochastic, asynchronous, nonlinear systems, often outperforming intuitive reasoning. Finally, in addition to discussing subtle numerical effects, small prediction discrepancies, and difficulties in generating the mapping between different abstractions levels, we conclude the paper by reviewing the intrinsic limitations of the current modeling methodology and by proposing a few suggestions for future work.
\end{abstract}

Keywords: swarm robotics, distributed control, swarm intelligence, microscopic and macroscopic modeling.

\section{Introduction}

In the last few years, distributed control principles have been successfully applied to a series of case studies in collective robotics: aggregation ${ }^{2,27,28}$ and segregation ${ }^{17}$, foraging ${ }^{21,33}$, collaborative stick pulling ${ }^{18,26}$, cooperative transportation ${ }^{7,10,22,36}$, flocking and navigation in formation $^{9,20,33}$, odor source localization ${ }^{14,16}$, cooperative mapping ${ }^{4,41}$, and soccer tournaments ${ }^{40}$. Each of these case studies used groups of robots or embodied simulated agents 
acting autonomously based on their own individual decisions. However, not all the architectures reported in these contributions were designed to control large numbers of robots. For instance, several approaches extensively exploit global communication capabilities $^{10,33,36,40,41}$, a characteristic which represents a bottleneck for the scalability of the collective system and may be, under certain environmental conditions, unfeasible. In other case studies, although the underlying principles of the control architecture were fully scalable, due to technical difficulties in experimentation with real robots, local explicit communication $^{14,4}$ or specific environmental information (e.g., nest energy ${ }^{21}$ ) was obtained with global communication, often combined with global positioning systems. While global positioning systems, depending on their specific implementation (e.g., standard GPS or the system used by Billard et al. ${ }^{4}$ ), do not necessarily prevent the scalability of the collective system, they require an additional level of sophistication of the individual unit in order to process the information broadcasted by the central reference device and are not always available in the target environment.

A possible paradigm for overcoming scalability issues and at the same time promoting robustness and individual simplicity is that proposed by an innovative computational and behavioral metaphor for solving distributed problems called Swarm Intelligence (SI) ${ }^{3,30}$. SI takes its inspiration from the biological examples provided by social insects ${ }^{5}$ such as ants, termites, bees, and wasps and by swarming, flocking, herding, and shoaling phenomena in vertebrates $^{37}$. The abilities of such natural systems appear to transcend the abilities of the constituent individual agents. In most biological cases studied so far, robust and coordinated group behavior has been found to be mediated by nothing more than a small set of simple local interactions between individuals, and between individuals and the environment. The SI approach emphasizes self-organization, distributedness, parallelism, and exploitation of direct (peer-to-peer) or indirect (via the environment) local communication mechanisms among relatively simple agents.

In addition to allowing for scalability, both statically (the control architecture can be kept exactly the same from a few units to thousands of units) and dynamically (units can be added or removed on the flight), the SI approach promotes robustness rather than efficiency. The resulting collective system is robust in facing a priori unknown environmental and team changes not only through unit redundancy but also through an adequate balance between exploitative and exploratory behavior, relying on an appropriate level of noise and number of mistakes in the coordination of the group. A natural example of this system behavior is represented by the foraging strategy of ants ${ }^{5}$. It is well known that recruitment processes based on trail-laying and -following mechanisms lead an ant colony to focus its current foraging power on a particular source of food (exploitation). What is, perhaps, less widely known is that not all the ants perfectly follow the trail left by teammates (or by themselves in a previous trip): a small percentage of them, depending on the ant species and on the environment in which it has evolved, leave the trail and, in doing so, allow the colony to discover new, possibly richer feeding opportunities close to the main path of the currently exploited source (exploration). Finally, a third advantage of the SI approach lies in the simplicity required at the individual level for achieving smart group behavior. Simplicity often increases the individual's robustness, allows for unit's miniaturization, reduces overall system cost, and represents a natural way to implement a sufficient amount of noise in coordination.

Probabilistic Modeling - The main motivation for developing a modeling methodology for swarm robotic systems is that, while SI principles are appealing from scalability, robustness, and individual simplicity point of view, they do not provide us with a way to quantitatively predict the swarm performance according to a particular metric or analyze 
further possible optimization margins and intrinsic limitations of this approach from an engineering point of view. In other words, if we want to achieve coordinated, self-organized group behavior based on local interactions, we need to have appropriate tools for understanding how to design and control individual units so that the swarm can achieve target behaviors and levels of performance. Models allow the engineer to capture the dynamics of these nonlinear, asynchronous, potentially large-scale systems at more abstract levels, sometimes achieving even mathematical tractability. More generally, modeling is a means for saving time, enabling generalization to different robotic platforms, and estimating optimal system parameters, including control parameters and number of agents in a team.

Although for a long period early in collective robotics research there was relatively little work in modeling of multi-robot systems, recently physicists and engineers have dedicated more attention to this problem (see, for example, related work performed by Kazadi et al. ${ }^{19}$, Lerman and Galstyan ${ }^{24}$, and Sugawara and Sano ${ }^{38,39}$ ). Moreover, modeling methodologies for swarm robotics systems must take into account mobility, local intelligence, intrinsic stochastic properties of the collective coordination based on SI-principles, and, potentially, several different modalities of interaction among individuals and between an individual and the environment (e.g., mechanical, electromagnetic, chemical). This extremely rich combination of system features has drastically reduced the applicability of modeling techniques developed and commonly used in other fields.

In this paper, we combine the expertise accumulated in building probabilistic microscopic ${ }^{18,27,28}$ and macroscopic models ${ }^{1,23,31,32}$ for distributed manipulation experiments characterized by different robotic platforms and tasks (aggregation, wall building, stick pulling) in a consistent framework. We believe that this work can be considered one of the first attempts to develop an ad hoc modeling methodology for swarm robotic systems. The strength of this research lies in the fact that, in contrast to contributions which did not aim to quantitatively correct predictions without free parameters ${ }^{19,23}$ and to those with fewer implementation levels ${ }^{24,38,39}$, we present here microscopic and macroscopic models and we validate them with real robots and/or embodied simulations, discussing in detail the mapping between different abstraction levels.

The Stick-Pulling Case Study - The experiment presented in this article is the follow-up of initial tests presented by Martinoli and Mondada ${ }^{26}$. The task is to locate sticks in a circular arena and to pull them out of the ground ${ }^{\mathrm{a}}$ using Khepera ${ }^{35}$ robots equipped with grippers and capable of distinguishing the sticks from walls and other robots with their frontal sensors. Due to the sticks' length, a single robot cannot pull a stick out of the ground alone; collaboration between two robots is necessary. As the robots have only local sensing capabilities and do not exploit a fully connected communication network, there is neither central nor global coordination among robots. Coordination is purely probabilistic and happens based on local interactions, strictly following the SI-principles mentioned above (see the experiment description in Section 2). Though all of the described experiments using real robots were conducted with teams ranging from one to six robots, only the cost of the equipment prevented them from being carried out with larger team sizes, as we did with the help of an embodied simulator and with our models.

The specific interest for the stick-pulling experiment lies in its strictly collaborative nature. In the class of distributed manipulation experiments we have considered thus far, the stick-pulling experiment is rather unique, since the other tasks studied can be completed by individual robots, the collective effort allowing the swarm mainly to improve its performance over time or robustness in task accomplishment. From a modeling point of view, two coupling

\footnotetext{
a Although the experiment is not intended to reproduce a biological system, the experiment presents several similarities with the extraction and transportation of matches performed by some ant colonies ${ }^{6}$.
} 
mechanisms among robots are overlapped in this experiment. First, robots modify a "shared blackboard" (i.e. the environment) and each robot's actions therefore indirectly influence those of its teammates, as in other distributed manipulation experiments. Second, robots trigger their mutual actions in a more direct way, following the precise temporal sequence required by the definition of a successful collaboration between pair of individuals (see Section 2).

Finally, it is worth noticing that a similar collaboration dynamics could require $r$-robots instead of two (see, for example, the description reported in Lerman et $a .^{23}$ ) and arise in a completely different experiment, one not involving object manipulation at all. For example, self-locomoted sensor nodes characterized by pseudo-random movement patterns, endowed with local communication capabilities, and engaged in a monitoring task over a welldelimited area, could be represented in the same abstracted way as robots engaged in the stick-pulling experiment. In this case, the metric used to assess the swarm performance could be related to the number of successful event detections reported by the swarm to a basestation knowing that, before emitting an alarm signal, at least $r$-nodes of the swarm should collectively agree to have detected the same event.

Research Contributions - This article aims to contribute to research in SI (i) by combining the previous contributions in microscopic and macroscopic modeling under a more rigorous, unified framework, (ii) by precisely describing the mapping between different implementation levels of the same experiment, (iii) by illustrating the application of the probabilistic modeling methodology with several examples of incremental complexity, and (iv) by investigating the strength and limitations of the current modeling methodology, particularly in comparison to other popular simulation tools such as sensor-based, embodied simulators.

\section{A Case Study: The Stick-Pulling Experiment}

In the case study described in this paper, robots must pull sticks out of the ground, an action that, due to the length of the sticks, requires the collaboration of two robots to be successful. The metric measured to quantitatively investigate and model the effects of variations of system parameters is the collaboration rate among robots, i.e., the number of sticks successfully taken out of the ground over time. The metric is nonspatial (i.e., we consider all the sticks pulled by the robots throughout the arena, independent of the sticks' placement) and measured by an external observer rather than by the robots themselves.

\subsection{The Physical Set-Up}

The experiment is carried out in a circular arena ( $40 \mathrm{~cm}$ of radius) delimited by a white wall. Four holes situated at the corners of a square with $30 \mathrm{~cm}$ edges hold white sticks $(15 \mathrm{~cm}$ long, diameter of $1.6 \mathrm{~cm}$ ) that, in their lowest position, protrude $5 \mathrm{~cm}$ above the ground (see Figure 1, left).

Groups of two to six Khepera robots, equipped with gripper turrets, are used to pull the sticks out of the ground. Because of their thinness, the sticks can be distinguished from the wall and from other robots using the Khepera's six frontal infrared proximity sensors. Because the sticks are too long to be pulled from the ground by a single robot's lifting motion, collaboration between two robots is required. After a successful collaboration, the stick taken out of the ground is released by the robot, and replaced in its hole by the experimenter. 

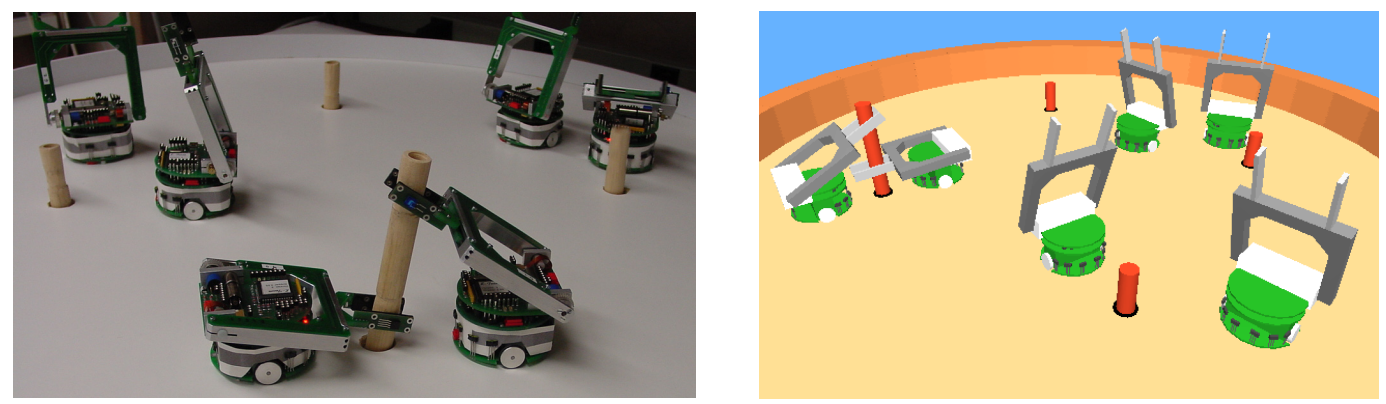

Figure 1 Left: Overview of the physical set-up for the stick-pulling experiments (4 sticks, arena of $40 \mathrm{~cm}$ in radius). Right: Corresponding set-up in the embodied simulator.

\subsection{Embodied Simulations}

In order to more systematically investigate the collaboration dynamics, we also implemented the experiment in Webots ${ }^{34}$, a 3D, kinematic, sensor-based simulator of Khepera robots (see Figure 1, right). Teams of two to 24 robots were simulated using Webots. The simulator computes trajectories and sensory input of the robots in an arena corresponding to a given physical set-up. The resulting simulation is sufficiently faithful for the controllers to be transferred to real robots without changes and for the simulated robot behaviors to be very similar to those of the real robots, as shown in several previous papers ${ }^{14,18,27,28}$. As speed-up ratio reference, a stick-pulling experiment using five robots takes on average 18 times less time if run with Webots on a Pentium III, $900 \mathrm{MHz}$ machine than if performed with real robots.

\subsection{The Robots' Controllers}

The behavior of a robot is determined by a simple hand-coded program that can be represented with a standard flow chart or a Finite State Machine (FSM), as depicted in Figure 2 , left. The behavioral granularity shown in Figure 2, left is arbitrary and is chosen by the experimenter so that the FSM captures all the details of interest. 

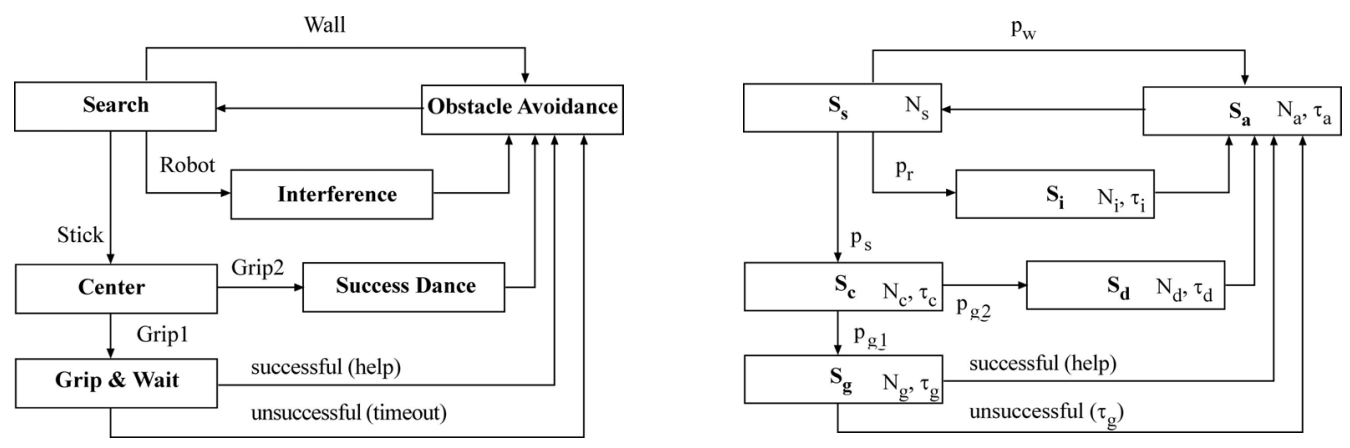

Figure 2 Left: FSM representing the robot controller. Transitions between states are deterministically triggered by sensory measurements. Right: PFSM representing an agent in the microscopic model or the whole robotic team in the macroscopic model. The parameters characterizing probabilistic transitions and states are explained in the text.

In addition to the default search behavior (moving in a straight line) and an obstacle avoidance behavior, the robot is endowed with a stick-gripping and -pulling procedure. The robot can determine from its arm elevation speed while pulling whether another robot is already gripping the same stick. While waiting for collaboration, another robot's attempt to lift the stick is similarly detected with the arm elevation sensor. If a single robot is holding the stick, we call such a grip a gripl. If another robot is already holding the stick and a searching robot finds and grips it, such a grip is called grip2. When a robot makes a grip1, it holds the stick raised half-way out of the ground and releases it when either the duration of the grip exceeds the gripping time parameter $\tau_{\mathrm{g}}$ (a failed collaboration), or another robot comes to make a grip2 (a successful collaboration). Once the stick is released, the robot turns away, performs obstacle avoidance for a few seconds, then returns to the search procedure. When a robot makes a grip2, the robot making the grip1 will release the stick, allowing its teammate to raise the stick completely. The robot making grip2 performs a short "success dance" (moving the arm up and down) to mark the successful collaboration, then releases the stick, performs obstacle avoidance for a few seconds, and resumes searching for sticks (see Extension 1).

Because of the way sticks are recognized (only by their thinness), a stick that is held by one robot can only be recognized when approached from the opposite side within a certain angle (approx. 125 degrees in the physical set-up). For the other angles of approach, both the stick and the robot are detected and the whole is taken for an obstacle. More details are reported in Ijspeert et al. ${ }^{18}$ but we note that the acceptable approach angle, expressed as a ratio $R_{g}$ over the whole approaching perimeter, is an important parameter in the collaboration dynamics of the system.

\section{Probabilistic Microscopic and Macroscopic Modeling}

The central idea of the probabilistic modeling methodology is to describe the experiment as a series of stochastic events with probabilities computed from the interactions' geometrical properties. The absolute location of the events on the arena is not considered in the models and the models' parameter calibration is achieved with systematic experiments using one or two real robots. Figure 2, right shows a Probabilistic Finite State Machine (PFSM) or Markov chain whose state-to-state transitions depend on the interaction probabilities of a robot with another teammate and with the environment. While in microscopic models each robot is represented by its own PFSM, in macroscopic models a single PFSM summarizes the whole robotic team, each of its states representing the average number of teammates in a particular 
state at a certain time step. In both types of models, the robots' PFSM(s) are then coupled with the environment. This coupling among robots via the environment (or in other experiments, direct peer-to-peer coupling, for example, through explicit communication) shapes the microscopic-to-macroscopic mapping, in particular determining its linear or nonlinear properties. Moreover, the environment can be considered as a passive, shared resource whose modifications are generated by the parallel actions of the robots. In order to compute the arbitrary nonspatial metric we are interested in (the collaboration rate), we keep track of each modification of the environment in the microscopic model or we estimate average environmental quantities in the macroscopic model.

The mean speed-up ratio for this experiment with five robots between the microscopic model (implemented in C) and Webots simulations is about $25^{\prime} 000$ on a Pentium III, $900 \mathrm{MHz}$ machine. That of the macroscopic model (currently implemented in Matlab ${ }^{\circledR}$ ) on the same machine is $4 \times N \times R, N$ being the total number of robots in the team and $R$ the total number of runs for obtaining the mean performance.

\subsection{Common Assumptions}

The current modeling methodology relies on two main, somewhat overlapping, common assumptions: spatial uniformity and the fulfillment of Markov properties, each of them briefly described in turn.

Nonspatial Models - The methodology relies on the assumption that the coverage of the arena by the groups of robots is as uniform as if the robots could hop around randomly on the surface. Robots' trajectories or specific robot spatial distributions therefore are not considered in the current models. We also assume that the absolute position of a given object to manipulate in the arena does not play a role: for instance, the object will have the same probability of being manipulated whether it is placed in the center or in the periphery of the arena.

Semi-Markov Models - We assume that the robot's future state depends only on its present state and on how much time it has spent in that state. This assumption is correct for a reactive robot controller extended with a time-out or following a predetermined sequence of actions (e.g., gripping a stick, dancing) that lasts a certain amount of time. The robots (and the environment) in the stick-pulling case study clearly obey this Markov property if we assume we are considering all robots' and environment's states of interest for computing the desired nonspatial metric (e.g., trajectory states - position and heading - can be neglected).

Both assumptions are valid in most of the experiments presented in this paper. In Subsection 6.2.1, we experimentally validate the nonspatial model assumption while in Subsection 6.2.3 we discuss instead an example of particular experimental constraints (overcrowded arena) where both assumptions are no longer valid.

\subsection{Models' Parameter Calibration}

The models presented in this paper are characterized by two different categories of parameters: transition probabilities and delays. In the following two subsections, we will describe how we calculated and measured all the parameters belonging to either one or the other category.

\subsubsection{Transition Probabilities}

Consistent with previous publications ${ }^{1,18,27,28,31,32}$, we compute the transition probabilities from a state to another based on simple geometrical considerations about the interaction (e.g., 
detection areas, approaching perimeters). The numerical values used for these geometrical parameters correspond to the average values measured in systematic tests with one or two real robots, as mentioned above. In nonspatial models, robots' positions on the arena are assigned randomly at each new iteration (or time step). At each iteration, the probability that a robot in the search mode will encounter a wall, a stick, or another robot is determined by their corresponding detection area divided by the whole arena area $A_{a}$. For instance, the probability of finding a stick can be computed as $p_{s}=A_{s} / A_{a}, A_{s}$ being the detection area of a stick. Similarly, $p_{w}=A_{w} / A_{a}$ and $p_{r}=A_{r} / A_{a}$ represent the probability of encountering a wall and another robot respectively. The total probability of encountering any other robot on the arena can be computed as $p_{R}=\left(N_{0}-1\right) p_{r}, N_{0}$ being the total number of robots in the arena. Additionally, since robots can perform a grip1 from any angle of approach, $p_{g l}=p_{s}$. On the other hand, since a stick available for grip2 can only be approached from a certain angle, as mentioned above, the probability of a grip2 event is $p_{g 2}=R_{g} p_{g l}$. The total number of sticks in the arena is $M_{0}$. See Ijspeert et al. ${ }^{18}$ for more details.

Furthermore, the current implementation of the model does not allow for overlapped areas of detection between objects of different type (e.g., robot, wall). Equation (1) expresses this limit mathematically:

(1) $p_{w}+p_{s} M_{0}+p_{R} \leq 1$

This, in turn, set a boundary for the maximal detection areas occupied by robots to $p_{R}=1$ $p_{w}-p_{s} M_{0}$. As we will see in subsection 6.2.3, this is just an approximation that forces additional robots to "squeeze" into their maximum available free space. In overcrowded scenarios, the modeling methodology reaches its limitations.

The numerical values used for the mean robot speed, the mean approaching angle for grip2, and the different mean detection radii of the objects are summarized in Table 1. These values are exactly the same as those reported in Ijspeert et al. ${ }^{18}$, although a single arena of 40 $\mathrm{cm}$ in radius was used at that time.

\begin{tabular}{|c|c|c|c|c|c|}
\hline $\begin{array}{c}\text { Mean robot } \\
\text { speed } \\
\mathrm{v}[\mathrm{cm} / \mathrm{s}]\end{array}$ & $\begin{array}{c}\text { Mean approaching } \\
\text { angle ratio } \\
\mathrm{R}_{\mathrm{g}}\end{array}$ & $\begin{array}{c}\text { Mean wall } \\
\text { detection distance } \\
\mathrm{R}_{\mathrm{w}}[\mathrm{cm}]\end{array}$ & $\begin{array}{c}\text { Mean seed } \\
\text { detection distance } \\
\mathrm{R}_{\mathrm{s}}[\mathrm{cm}]\end{array}$ & $\begin{array}{c}\text { Mean robot } \\
\text { detection distance } \\
\mathrm{R}_{\mathrm{r}}[\mathrm{cm}]\end{array}$ & $\begin{array}{c}\text { Arena } \\
\text { Radius } \\
\mathrm{R}_{\mathrm{a}}[\mathrm{cm}]\end{array}$ \\
\hline 8 & 0.35 & 6 & 6.4 & 10 & $\begin{array}{c}40 \text { to } \\
5000\end{array}$ \\
\hline
\end{tabular}

Table 1: Parameters used in the models of the stick-pulling experiment. Detection distances are meant from the center of the robot to the center of the object detected.

\subsubsection{Time Discretization and Delays}

The current probabilistic methodology generates time-discrete models. In this subsection we would like to motivate our choice and explain how we establish the discretization interval $T$ as well as the discretized delays considered in the models.

Time-Discrete vs. Time-Continuous Models - At a first glance, we might think that, since the physical set-up operates in continuous, real time and agents operate asynchronously, the best way to obtain a faithful model would also be to use a time-continuous model. As usual, we would emulate continuous time in simulation by choosing a small time step combined with a standard numerical integration algorithm (e.g., Runge-Kutta). However, if we look more closely, we realize that the description of the system we are interested in (see Figure 2, right) is at a much higher level than that which would involve mass, speed, forces, positions, and so on: it is a description characterized by logical operators and behavioral states. Robots are natural hybrid systems, in a strictly automatic control sense: they consist of time- 
continuous elements (e.g., motors, sensors, and analog electronics) but are also endowed with microcontrollers that, in addition to being digital and clocked, may also implement high-level, logical control operations. The success dance in the stick-pulling experiment is a typical example: its duration is triggered by a sensory stimulus and systematically lasts until an incremental timer of the robot reaches a pre-established timeout. Since we are not interested in modeling the details of the success dance, a time-discrete model would capture this duration precisely without extra computation in between.

This is the main reason we believe time-discrete models are the most adequate solution for the level of description we are aiming to, although at microscopic level they will force state transitions of the PFSMs to happen at the end of time steps rather than completely asynchronously. Emulation of time continuity would simply add simulation time (even macroscopic models, being, in general, nonlinear, must be solved numerically) without increasing prediction accuracy and prevent the description of microscopic and macroscopic models under the same framework.

Time Discretization Interval - Consistent with previous publications ${ }^{1,18,27,28,31,32}$, each iteration of our models corresponds to a time step of a finite duration in real time. The duration of a time step is equivalent to the time needed for a robot, moving with a certain mean speed $v$ and having a certain mean detection width $w_{i}$ for the smallest object in the arena $i$ (in our case, a stick), to cover the smallest object's detection area. Equation (2) shows how to compute the duration $T$ of one time step in the modeling methodology:

$$
T=\frac{A_{s}}{v w_{s}}=\frac{\pi R_{s}^{2}}{v 2 R_{s}}=\frac{\pi R_{s}}{2 v}
$$

Choosing the smallest object is a way to ensure that the time granularity is high enough to capture every occurrence of the system's fastest detection event. This is an approximation which links time partitioning with probability-space partitioning and is consistent with the fact that our models are nonspatial ${ }^{b}$. Although this method for choosing the time step is completely heuristic, it has provided, combined with the method of calculating the transition probabilities explained in Subsection 3.2.1, the best results so far not only in the stick-pulling experiment but also in aggregation ${ }^{28}$ and wall-building ${ }^{27,28}$ experiments performed with different robotic platforms. We will discuss the difficulties inherent to parameter calibration and time discretization more extensively in Subsection 6.2.2.

Measured and Discretized Delay Values -Table 2 summarizes the values of delays used in all the models presented in this paper. The measured mean delays are exactly the same used in Ijspeert et al. ${ }^{18}$ The time step has been calculated with Eq. (2): $T=1.26 \mathrm{~s}$. Of course, any gripping time parameter $\tau_{g}$ will be also discretized to $T_{g}$ iterations in the models ${ }^{\mathrm{c}}$.

\begin{tabular}{|c|c|c|c|c|}
\hline Delay & Centering & Success Dance & Obstacle Avoidance & Interference \\
\hline Mean measured value [s] & $\tau_{\mathrm{c}}=10$ & $\tau_{\mathrm{d}}=6$ & $\tau_{\mathrm{a}}=1$ & $\tau_{\mathrm{i}}=2$ \\
\hline Discretized value [iterations] & $\mathrm{T}_{\mathrm{c}}=8$ & $\mathrm{~T}_{\mathrm{d}}=5$ & $\mathrm{~T}_{\mathrm{a}}=1$ & $\mathrm{~T}_{\mathrm{i}}=2$ \\
\hline
\end{tabular}

Table 2: Duration of the different robot maneuvers described in subsection 2.3.

\footnotetext{
${ }^{\mathrm{b}}$ An alternative explanation on how to come to this heuristic formula can be found in Martinoli's Ph.D. thesis ${ }^{29}$, chapter 4 . In Ijspeert et al. ${ }^{18}$ we used a slightly different robot detection width (a value which can be considered as a weighted sum of all the different detection widths specific to different objects in the arena).

${ }^{c}$ As the time step is different from Ijspeert et al. ${ }^{18}$, the corresponding discretized delays used in the models of this paper may be slightly different after rounding.
} 


\section{Examples of Models Characterized by two States}

Before describing implementation details and results of the full-system model, we introduce two examples of 2-state PFSMs. Both examples not only represent key sub-chains of the full system's Markov chain we will describe in Section 5 but also can be considered as basic elements constituting models of other swarm robotic experiments generated with the methodology presented in this paper.

Indeed, the first example is concerned with a delay component affecting each robot's behavior. The delay could be defined by an internal timer (e.g., the success dance or any finite period required for processing information) or by a specific interaction with the environment (e.g., gripping a stick or any finite period needed for sensing or acting in the environment) or a teammate (e.g., obstacle avoidance or any finite period needed for information broadcast). The corresponding Difference Equations (DEs) at the macroscopic level are linear since we assume that there is no coupling between agents, neither directly (agent-to-agent) nor indirectly (through the environment): the specific action generating the delay lasts always for a pre-established period independently from the state of the system.

The second example can instead be considered a simplified model of the stick pulling experiment which already involves the overlapping of the two nonlinear coupling mechanisms - among the agents and therefore among the DEs - mentioned in Section 1: environmental modification and triggering of mutual actions. In contrast to other distributed manipulation experiments concerned with, for instance, aggregation and segregation of objects based on stigmergic $^{\mathrm{d}}$ mechanisms, the environmental modification in the stick pulling experiment does not have plastic properties as result of the action of a single robot. In fact, as soon as a robot releases a gripped stick because no other robots came to help, the environmental modification performed (i.e., pulling the stick halfway out) is automatically reversed by the force of gravity (i.e., the stick falls back into its hole). Furthermore, in the version of the stick pulling experiment presented in this paper the triggering of mutual actions is obtained by the sequence of movements encoded in each robot controller, in essence, a primitive handshaking protocol that uses the stick as the communication medium. Nothing will prevent us, however, from implementing this handshaking protocol with local wireless communication and obtaining, at the model level, the same description, with states simply characterized by different delays.

Unless otherwise stated, experiments using the microscopic model have been repeated 100 times and error bars represent standard deviation among runs. At the macroscopic level, of course, one run suffices, since only central tendencies (in our case the mean swarm performance) can be predicted.

\subsection{Search and Obstacle Avoidance States}

A first key element of the full-system model representing the stick-pulling experiment is a delay state. A delay state simply represents a behavior the robot will perform for a certain duration $T_{a}$ with a probability $p_{a}$. The probability of leaving the delay state after $T_{a}$ is one and is independent of the robot's interaction with the environment or with the teammates. As an example for a delay state in the stick pulling experiment, we have chosen a sub-chain of the system represented by the default search state coupled with an obstacle avoidance state. In reality, depending on the obstacle type, an avoidance maneuver may be characterized by different durations and different probabilities of performing it. Furthermore, depending on the

\footnotetext{
${ }^{\mathrm{d}}$ The concept of stigmergy was introduced for the first time by P.P. Grassé ${ }^{12}$ : it comes from the Greek stigma (sting) and ergon (work); it describes a phenomenon in which a plastic modification of the environment introduced by the work of an individual is perceived as a stimulating configuration by other agents (or by the same agent at later time).
} 
coverage of the proximity sensors and the collision angle between two robots, we may or may not have obstacle avoidance behavior mutually triggered (and therefore a nonlinear coupling in the DEs as shown in Lerman and Galstyan ${ }^{24}$ ). For sake of simplicity, in this subsection we will use a single obstacle type and no mutual triggering of obstacle avoidance ${ }^{\mathrm{e}}$. Values for probabilities and durations are derived from Table 1 and Table 2.

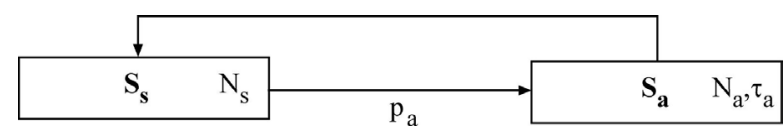

Figure 3: A simple sub-chain consisting of a search and an obstacle avoidance state. The numerical values used in this example have been derived from the values of Table 1 and Table 2 using parameters for a generic obstacle: $T_{a}=2$ iterations and $p_{a}$ is a function of the set-up (for example, $p_{a}=0.63$ for the mean probability of encountering an obstacle in an arena of $40 \mathrm{~cm}, 4$ robots, and 4 sticks; in this case teammates, walls, and sticks are considered all as obstacles).

Figure 3 graphically represents the state diagram of this simple PFSM. $N_{s}$ and $N_{a}$ represent the numbers of robots in the search state and obstacle avoidance states respectively. In the microscopic model, these are binary values at the level of each PFSM since an agent can be in one single state at the time; they are integer values corresponding to the actual numbers of robots in each state at the team level. In the macroscopic model instead, $N_{s}$ and $N_{a}$ are real values representing the average numbers of robots in a certain state, at a given time step, and over multiple runs.

Since the state variables of the macroscopic model are represented by continuous quantities, the PFSM of Figure 3 representing the whole swarm can be described by the following DE system:

(3) $\quad N_{s}(k+1)=N_{s}(k)-p_{a} N_{s}(k)+p_{a} N_{s}\left(k-T_{a}\right)$

(4) $\quad N_{a}(k+1)=N_{0}-N_{s}(k+1)$

$k=0,1,2 \ldots$ represents the current iteration. $N_{x}(k)$ represents the value of the state variable $N_{x}$ at time $k T$; the notation $N_{x}(k)$ instead of $N_{x}(k T)$ is standard in the automatic control literature. Equation (3) states that the average number of robots in the search state at iteration $k+1$ is equal to the average number of robots in search at iteration $k$ minus those which left for an obstacle avoidance maneuver plus those which have terminated their avoidance. Equation (4) simply exploits the conservation of the total number of robots for calculating the average number of robots in obstacle avoidance.

It is worth mentioning that, unless otherwise stated, we assume that no robots exist before $k=0$ (a standard convention for this type of time-delayed DE). Mathematically speaking:

(5) $\quad N_{s}(k)=N_{a}(k)=0 \quad$ if $\quad k<0$

The initial conditions for the DE system are $N(0)=\left[\begin{array}{lll}N_{s}(0) & N_{a}(0)\end{array}\right]^{\mathrm{T}}=\left[\begin{array}{ll}N_{0} & 0\end{array}\right]^{\mathrm{T}}$ (all robots are in search state at the beginning of the experiment).

\footnotetext{
${ }^{\mathrm{e}}$ As shown in Subsection 6.2, this linear approximation is quite faithful for a platform such as the Khepera robot which is endowed with a proximity sensory belt affected by relevant blind angles on the sides, around the wheels. Namely, this implies in turn that, depending on the angles of approach, an interaction between robots may involve only one of them exhibiting obstacle avoidance behavior.
} 


\subsubsection{Microscopic and Macroscopic Results}

Figure 4, left shows a comparison between microscopic and macroscopic predictions for the mean number of searching robots at steady state and different team sizes. As it could be easily demonstrated mathematically by summing up and averaging individual quantities, in a linear system the microscopic-macroscopic transformation is straightforward and no approximation is required for obtaining a closed form. As a consequence, both models' predictions perfectly coincide on the stationary mean number of robots in search, also for small teams, while the standard deviation among runs and over time of the microscopic model is about inversely proportional to the swarm size. It is worth noting that the mean number of robots in obstacle avoidance at steady state can be easily derived with Eq. (4).
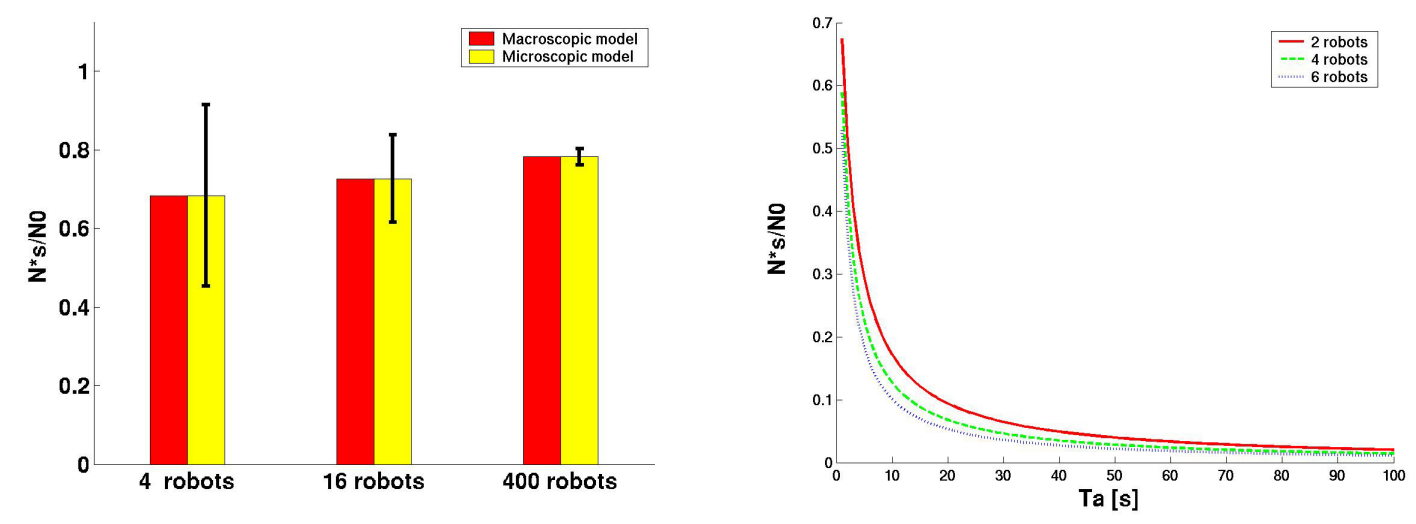

Figure 4: Left: Comparison of the steady state $N_{s}^{*}$ obtained with microscopic and macroscopic models for different team sizes and different arenas (density of teammates per surface unit was kept constant). The height of the microscopic column represents the mean value of $N_{s}^{*}$ over a 1000 $\mathrm{s}$ time window and over 100 runs. The slight increase in the mean value for different team sizes is due the fact that the wall detection surface becomes proportionally smaller in bigger arenas. The error bars represent a mean standard deviation calculated as an average of the standard deviations measured on each run over the same time window. Right: Graphical representation of Eq. (7) for different delay durations and different probabilities of encountering an obstacle in the $40 \mathrm{~cm}$ arena (the greater the swarm size, the larger $p_{a}$ is).

\subsubsection{Steady State Analysis}

Since the DE system (3)-(4) is linear, we can analyze the steady state of the system either using a z-transform (frequency domain) or in time domain. Both analyses bring to the same result.

Frequency domain - Equation (3) can be transformed using the right shift and left shift theorems in the z-space as follows:

(6) $z N_{s}(z)-z N_{0}=N_{s}(z)-p_{a} N_{s}(z)+p_{a} N_{s}(z) z^{-T_{a}}$

Solving for $N_{s}(z)$ and applying the limit theorem we obtain:

(7) $N_{s}^{*}=\lim _{k \rightarrow \infty} N_{s}(k)=\lim _{z \rightarrow 1}(z-1) N_{s}(z)=\frac{N_{0}}{1+p_{a} T_{a}}$ 
And therefore through the robots' conservation law:

(8) $\quad N_{a}^{*}=N_{0}-N_{s}^{*}=\frac{N_{0} p_{a} T_{a}}{1+p_{a} T_{a}}$

$N^{*}=\left[\begin{array}{ll}N_{s}^{*} & N_{a}^{*}\end{array}\right]^{\mathrm{T}}$ represents the state vector of the DE system in the steady state regime.

Time domain - Equation (4) could have been written also in the same form as Eq. (3), i.e., as a DE instead of an equation:

(9) $\quad N_{a}(k+1)=N_{a}(k)+p_{a} N_{s}(k)-p_{a} N_{s}\left(k-T_{a}\right)$

Equation (9) represents a delay state. A first, intuitive step for calculating the steady state of this equation could be to set $N_{a}(k+1)=N_{a}(k)=N_{a}^{*}$ and $N_{s}(k)=N_{s}\left(k-T_{a}\right)=N_{s}^{*}$. However, we can immediately see that in this case Eq. (9) will be underdetermined, i.e., $0=0$.

A work-around step for this problem is as follows: we can say that after $T_{a}$ iterations, every robot that enters the delay state must leave it with probability one. Since no robots exist for negative iterations (see Eq. (5)), the only time during which $N_{a}$ can be increased is during the first $T_{a}$ iterations (full inflow with probability $p_{a}$ and zero outflow). Although $N_{s}(k)$ may vary during the first $T_{a}$ iterations, we can approximate it as a constant (i.e., as it was in steady state) if $T_{a}$ is small:

(10) $N_{a}^{*}=\int_{0}^{T_{a}} p_{a} N_{s}^{*} d k=p_{a} N_{s}^{*} \int_{0}^{T_{a}} d k=p_{a} N_{s}^{*} T_{a}$

By combining Eq. (10) with the robots' conservation law is easy to demonstrate that $N_{s}^{*}$ and $N_{a}^{*}$ can be expressed as we calculated via the z-transformation (Eq.(7) and (8)). This second method of calculating the steady state vector for delay states is extremely useful if the whole system involving this type of state is nonlinear. This will be the case in the full-system model describing the dynamics of the stick-pulling experiment. Figure 4, right shows graphically how the normalized average number of searching robots at the steady state is influenced by the time delay $T_{a}$ and different probabilities $p_{a}$.

\subsection{Search and Grip States}

A second key sub-chain of the full-system model captures the two nonlinear couplings among agents mentioned before: environmental modification and triggering of mutual actions. The former mechanism, although it does not generate plastic modifications of the environment, has indirect consequences on the action of the teammates since the gripping of a stick reduces the opportunities for other teammates to find free sticks for grip1. The latter mechanism is instead due to the strictly collaborative nature of the stick-pulling experiment and the way robots communicate through sticks' manipulation.

If we look carefully the PFSM of Figure 2, we can notice that several states can be thought as simple delay states. The durations of the delays do not exceed a few seconds and are therefore much shorter than most of the values of the gripping time parameter considered in this paper (up to $600 \mathrm{~s}$ ). As a consequence, not only does the sub-chain described in this section represent an example of modeling swarm robotic experiments that involve both distributed manipulation and local communication, it also represents the core of the collaboration dynamics in the stick-pulling experiment. Indeed, by neglecting all the minor delays, the full-system model reduces to the very same two states: search and grip (see Figure $5)$. 


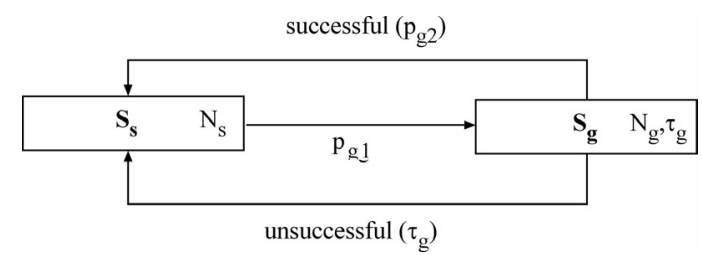

Figure 5: The key sub-chain representing the dynamics of collaboration in the stick-pulling experiment. The numerical values used in this simplified model have been derived using Table 1.

The following system of DEs represents the macroscopic model for this search-grip subchain:

(11) $N_{s}(k+1)=N_{s}(k)-\Delta_{g 1}(k) N_{s}(k)+\Delta_{g 2}(k) N_{s}(k)+\Delta_{g 1}\left(k-T_{g}\right) \Gamma\left(k-T_{g} ; k\right) N_{s}\left(k-T_{g}\right)$

(12) $N_{g}(k+1)=N_{0}-N_{s}(k+1)$

In words, Eq.(11) tells us that the mean number of robots in search state at any time is decreased by the robots transitioning to a gripping state $\left(\Delta_{g 1}\right)$ and is increased by the robots coming back from a successful collaboration $\left(\Delta_{g 2}\right)$ and those coming back from an unsuccessful collaboration $\left(\Delta_{g 1} \Gamma\right)$. Eq. (12) again exploits the conservation of the total number of robots for calculating the mean number of robots in the grip state.

The nonlinear coupling between equations is achieved with $\Delta$ - and $\Gamma$-functions as follows:

(13) $\Delta_{g 1}(k)=p_{g 1}\left[M_{0}-N_{g}(k)\right]$

(14) $\Delta_{g 2}(k)=p_{g 2} N_{g}(k)$

(15) $\Gamma\left(k-T_{g} ; k\right)=\prod_{j=k-T_{g}}^{k}\left[1-p_{g 2} N_{s}(j)\right]$

$\Delta_{g 1}$-function characterizes the environmental modification mechanism for single robots and the $\Delta_{g 2}$ and $\Gamma$-functions characterize the triggering/non-triggering of mutual actions. Eq. (13) indicates the number of sticks free for gripping is equal to the total number of sticks $M_{0}$ minus those that are already "busy". Eq. (14) tells us that we need another robot already gripping a stick and a correct approaching angle for the searching robot in order to achieve successful collaboration while Eq. (15) represents the fraction of robots that abandon the grip state after the time spent in this state exceeds their gripping time parameter $T_{g}$. As explained more extensively in Lerman et al. ${ }^{23}$, this is equivalent to calculating the probability that no other robot came "to help" during the time interval $\left[k-T_{g}, k\right]$.

Finally, our team metric, the (average) collaboration rate $\bar{C}_{t}$, can be computed from the cumulative number of successful collaborations $C$ over the maximal number of iterations $T_{e}$ :

(16) $C(k)=p_{g 2} N_{s}(k) N_{g}(k)$

(17) $\bar{C}_{t}=\frac{\sum_{k=0}^{T_{e}} C(k)}{T_{e}}$ 
As in the case of the 2-state system described in Section 4.1, the initial conditions for the DE system are $N(0)=\left[\begin{array}{ll}N_{0} & 0\end{array}\right]^{\mathrm{T}} \quad$ (all robots are in search state at the beginning of the experiment).

\subsubsection{Microscopic and Macroscopic Results}

Figure 6 shows a comparison between microscopic and macroscopic predictions of the collaboration rate (left column) and of the steady state number of robots in search (right) for different arenas and swarm sizes. A first striking result is the existence of two different system dynamics as a function of the ratio between number of robots and number of sticks. When there are more robots than sticks, the collaboration rate increases monotonically with the gripping time parameter and eventually saturates in a plateau corresponding to the optimal collaboration rate. In other words, under these conditions, it is a good strategy for a robot gripping a stick to wait a very long time for another robot to help, because there will always be at least one "free" robot available. In contrast, when there are fewer robots than sticks, waiting a very long time becomes a poor strategy, because all the robots lose time holding different sticks while no other robots are available to collaborate. As an extreme example, an infinite gripping time parameter would lead to a null collaboration rate with all robots eventually holding a different stick permanently. Although this intuitive explanation is correct for the physical set-up we considered in this paper, it is not generally true since the sticks-torobots ratio at which the system bifurcates is dependent on the $R_{g}$ parameter, as we will show in the next subsection and as we have also demonstrated in the full-system model in a recent publication $^{32}$.

A second observation we can make about Figure 6 is that, much like the 2-state example presented in subsection 4.1, the smaller the swarm size is, the larger the standard deviation is among runs using the microscopic model (visible in both the collaboration rate and steady state variables). Here we notice, however, that for very small swarm sizes for which there are at most the same number of robots as sticks, (see Figure 6, left, first row) macroscopic and microscopic models' predictions diverge quantitatively. Other, much smaller discrepancies can be observed between microscopic and macroscopic predictions with larger swarm sizes (400 and 600 robots, Figure 6, last row). This is counterintuitive since one would think that the problem shown in the first row of Figure 6 would only have to do with the small number of robots and sticks used in this scenario, quantities not large enough to satisfy the law of the large numbers on which macroscopic models base their calculations of central tendencies. A more careful inspection of the state variables in steady state, much like what was done in Subsection 4.1.1, reveals that the collaboration rate is not correctly predicted when, at the source, the steady state variables used for calculating it are different (see Figure 6, right column). In the case of nonlinear systems, we can no longer simply add and average equations defining individual agent's PFSMs for obtaining the PFSM representing the whole team. Approximations for obtaining closed formulas representing the macroscopic PFSM depend on the specific nonlinearity involved in the coupling and this, in combination with the fact that in smaller swarm sizes, the continuous quantities of the state variables representing the macroscopic level are in stronger contrast with the integer quantities representing the microscopic level, generates discrepancies between these two types of models. A quantitative analysis of the discrepancies of prediction between microscopic and macroscopic models is beyond the scope of this paper (see Section 6 for further discussion on this point). 

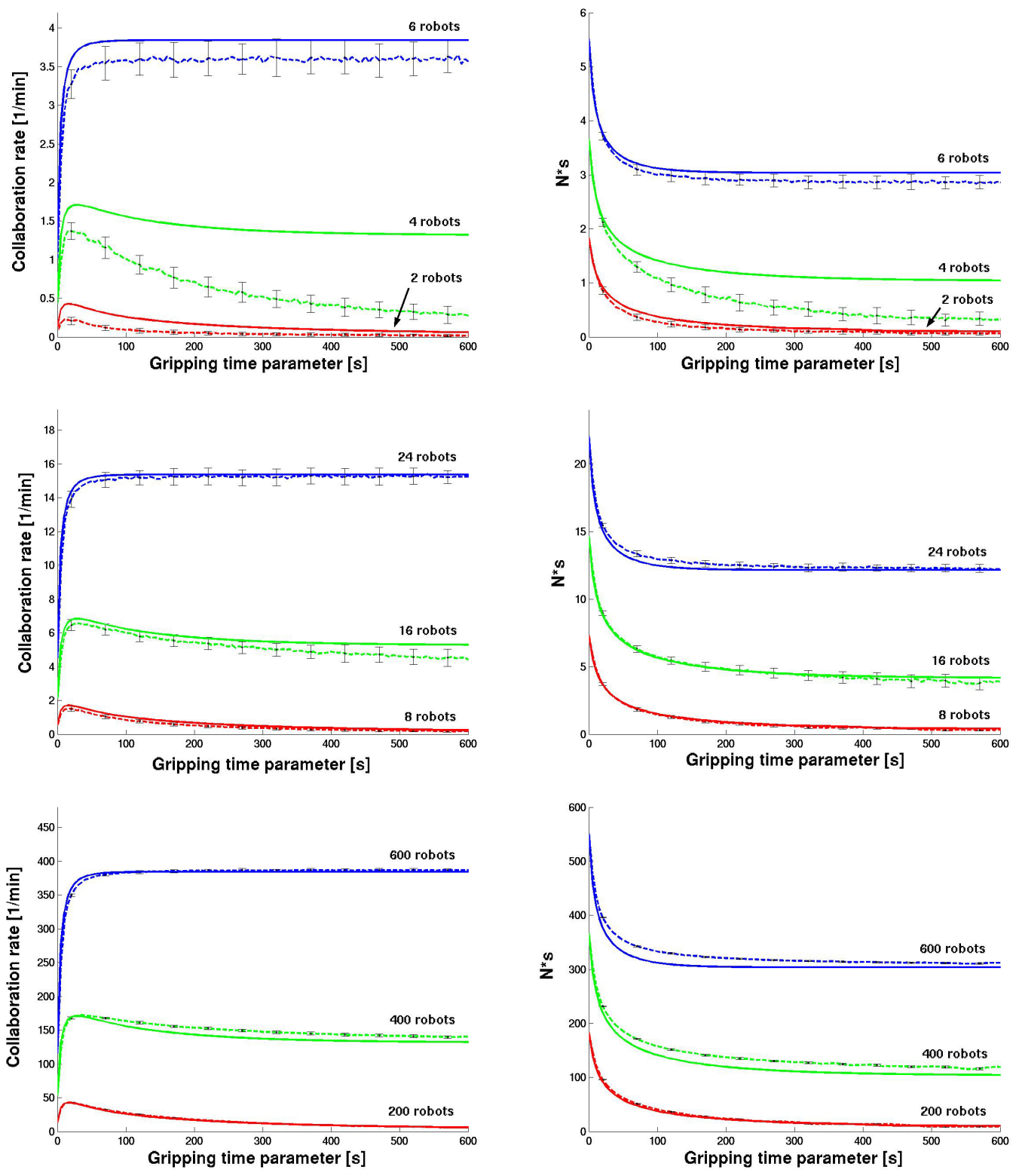

Figure 6: Comparison between microscopic and macroscopic predictions for the simplified stick-pulling model depicted in Figure 5. The density of robots and sticks in each arena are invariant. Rows one to three represent results obtained in arenas of 40,80 , and $400 \mathrm{~cm}$ in radius respectively. Left column: the collaboration rate as a function of the gripping time parameter for different swarm sizes. Right column: the steady state values of the average number of robots in the search state for different swarm sizes. Steady state values have been calculated over a window of $1000 \mathrm{~s}$ after at least $5^{*} \tau_{\mathrm{g}} \mathrm{s}$ from the beginning of the experiment. The error bars represent a mean standard deviation calculated as an average of the standard deviations measured on each run over the $1000 \mathrm{~s}$ time window. 


\subsubsection{Steady State Analysis}

Since the system of DEs (11)-(15) is nonlinear, we cannot analyze it in z-space, as we did with the previous 2-state sub-chain. Much as Lerman et al. have proposed for a timecontinuous version of the same sub-chain ${ }^{23}$, we perform a steady state analysis of the system in time domain.

By setting $N_{s}(i)=N_{s}^{*}$ and $N_{g}(i)=N_{g}^{*}$ for all $i$ between $k-T_{g}$ and $k+1$ in Eqs. (11)-(16) and substituting Eqs. (13) and (14) in Eq. (11), we obtain:

(18) $0=-p_{g 1}\left(M_{0}-N_{g}^{*}\right) N_{s}^{*}+p_{g 2} N_{g}^{*} N_{s}^{*}+p_{g 1}\left(M_{0}-N_{g}^{*}\right) N_{s}^{*} \Gamma^{*}$

(19) $N_{g}^{*}=N_{0}-N_{s}^{*}$

(20) $\Gamma^{*}=\left(1-p_{g 2} N_{s}^{*}\right)^{T_{g}}$

(21) $C^{*}=p_{g 2} N_{s}^{*} N_{g}^{*}$

First, we determine when the number of collaborations is maximized as a function of the number of robots in the search state (or in the grip state, respectively). To do this, we insert Eq. (19) in Eq. (21), perform a partial derivative over $N_{s}^{*}$ and set the result equal to zero. $C^{*}$ is maximal when $N_{s}^{*}=N_{0} / 2$.

By inserting this result, $p_{g 2}=R_{g} p_{g 1}$, Eqs. (19) and (20) in Eq. (18), we obtain the following transcendental equation:

(22) $0=-\left(M_{0}-\frac{N_{0}}{2}\right)+R_{g} \frac{N_{0}}{2}+\left(M_{0}-\frac{N_{0}}{2}\right)\left(1-p_{g 1} R_{g} \frac{N_{0}}{2}\right)^{T_{g}^{o p t}}$

Introducing $\beta=N_{0} / M_{0}$ and solving the equation for $T_{g}^{\text {opt }}$, we obtain:

(23) $T_{g}^{\text {opt }}=\frac{1}{\ln \left(1-p_{g 1} R_{g} \frac{N_{0}}{2}\right)} \ln \frac{1-\frac{\beta}{2}\left(1+R_{g}\right)}{1-\frac{\beta}{2}}$

Equation (23) tells us that an optimal $T_{g}$ exists if all the arguments of the logarithms are greater than zero. While this condition is met for the first logarithm in all our scenarios, the argument of the second logarithm depends on $\beta$ and $R_{g}$. It can be demonstrated ${ }^{\mathrm{f}}$ that an optimum exists if and only if:

(24) $\beta<\beta_{c}=\frac{2}{1+R_{g}}$

For all other cases, the collaboration rate is a monotonically increasing and eventually saturating function of $T_{g}$. Figure 7 graphically demonstrates the meaning of Eq. (24).

\footnotetext{
f While for $2 /\left(1+R_{g}\right) \leq \beta \leq 2$ the second logarithm of Eq. (23) does not exist, more generally, if $\beta \geq 2 /\left(1+R_{g}\right), N_{s}^{*}$ in Eq. (18) will be always greater than $N_{0} / 2$, i.e., a not optimal value. This can be easily demonstrated by introducing $\beta=N_{0} / M_{0}$ in Eq. (18), solving for $\beta$ and comparing with Eq. (24).
} 

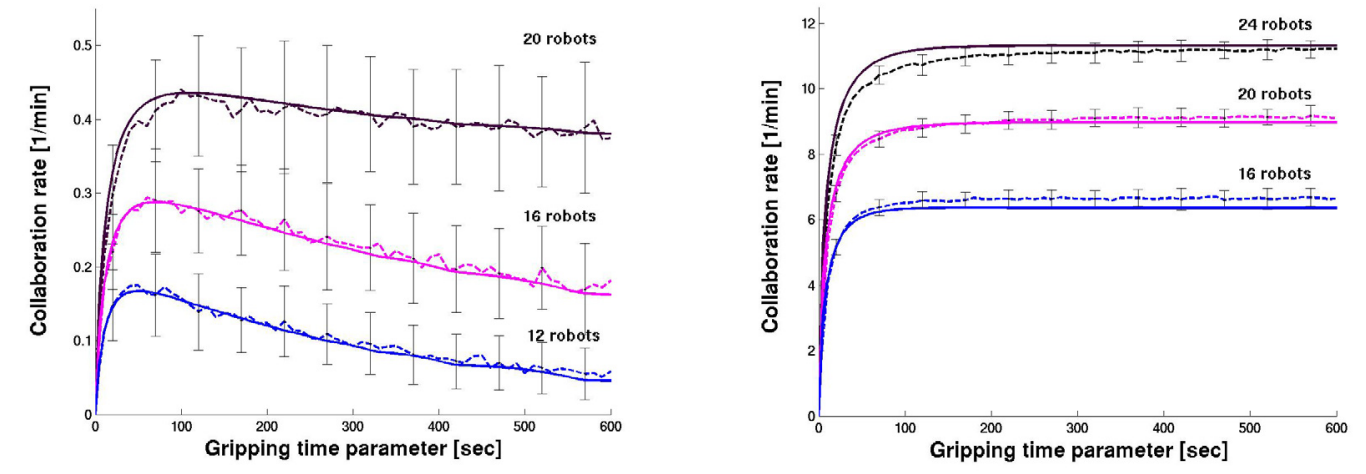

Figure 7: Graphical illustration of Eq. (24) for an arena of $80 \mathrm{~cm}$ and 16 sticks (microscopic and macroscopic predictions overlapped). Left: $\beta<2 /\left(1+R_{g}\right)$, with $R_{g}=0.035$. Right: $\beta \geq 2 /\left(1+R_{g}\right)$ with $R_{g}=1.0$.

Equation (24) tells us that the bifurcation of the system (optimal $T_{g}$ is found vs. no optimum exists) is a function of the collaboration parameter $R_{g}$. Notice that, for instance, if the collaboration is very difficult (i.e., $R_{g}$ is very small), there could be situations where although we have a greater number of robots than sticks (see Figure 7, left, 20 robots line), the optimal collaboration rate may still be achieved only with a specific $T_{g}$. In other words, when it is difficult to collaborate, in order to enhance the number of collaborations, it is worth abandoning the sticks after a while and probabilistically increasing the critical mass of robots working in another area of the arena. Although the precise team size at which the bifurcation happens in the real system cannot be correctly computed with Eq. (24), this equation allows us to better situate intuitive considerations such as those presented in Subsection 4.2.1.

\section{Full-System Model}

Having introduced the two key sub-chains characterizing the dynamics of the stickpulling experiment, we are now ready to analyze the full-system model. We can already imagine that the description of the full system at the macroscopic level will involve a system of nonlinear, coupled, time-delayed DEs.

As mentioned above, Figure 2, right shows the PFSM of the full system. The macroscopic model of the full system can be formalized as follows ${ }^{\mathrm{g}}$ :

$$
\begin{aligned}
N_{s}(k+1)= & N_{s}(k)-\left[\widetilde{\Delta}_{g 1}(k)+\Delta_{g 2}(k)+p_{w}+p_{R}\right] N_{s}(k)+\widetilde{\Delta}_{g 1}\left(k-T_{c g a}\right) \Gamma\left(k-T_{g a} ; k-T_{a}\right) N_{s}\left(k-T_{c g a}\right) \\
& +\Delta_{g 2}\left(k-T_{c a}\right) N_{s}\left(k-T_{c a}\right)+\Delta_{g 2}\left(k-T_{c d a}\right) N_{s}\left(k-T_{c d a}\right)+p_{w} N_{s}\left(k-T_{a}\right)+p_{R} N_{s}\left(k-T_{i a}\right) \\
N_{a}(k+1)= & N_{a}(k)+\widetilde{\Delta}_{g 1}\left(k-T_{c g}\right) \Gamma\left(k-T_{g} ; k\right) N_{s}\left(k-T_{c g}\right)+\Delta_{g 2}\left(k-T_{c}\right) N_{s}\left(k-T_{c}\right)+\Delta_{g 2}\left(k-T_{c d}\right) N_{s}\left(k-T_{c d}\right) \\
& +p_{w} N_{s}(k)+p_{R} N_{s}\left(k-T_{i}\right)-\widetilde{\Delta}_{g 1}\left(k-T_{c g a}\right) \Gamma\left(k-T_{g a} ; k-T_{a}\right) N_{s}\left(k-T_{c g a}\right) \\
& -\Delta_{g 2}\left(k-T_{c a}\right) N_{s}\left(k-T_{c a}\right)-\Delta_{g 2}\left(k-T_{c d a}\right) N_{s}\left(k-T_{c d a}\right)-p_{w} N_{s}\left(k-T_{a}\right)-p_{R} N_{s}\left(k-T_{i a}\right)
\end{aligned}
$$

(27) $N_{i}(k+1)=N_{i}(k)+p_{R} N_{s}(k)-p_{R} N_{s}\left(k-T_{i}\right)$

\footnotetext{
$\mathrm{g}$ The system of DEs presented in this paper is consistent with the description presented in previous publications $^{31,32}$ with one exception: the number of sticks available for grip1 as been here decreased by a factor $N_{d}$ since actually, as explained in subsection 2.3, the experimenter replaces the stick in the hole only when the success dance of the robot has terminated. Although the collaboration rate changes only minimally with this correction, the current model is more faithful to the real experiment.
} 
(28) $N_{c}(k+1)=N_{c}(k)+\widetilde{\Delta}_{g 1}(k) N_{s}(k)+\Delta_{g 2}(k) N_{s}(k)-\widetilde{\Delta}_{g 1}\left(k-T_{c}\right) N_{s}\left(k-T_{c}\right)-\Delta_{g 2}\left(k-T_{c}\right) N_{s}\left(k-T_{c}\right)$

(29) $N_{d}(k+1)=N_{d}(k)+\Delta_{g 2}\left(k-T_{c}\right) N_{s}\left(k-T_{c}\right)-\Delta_{g 2}\left(k-T_{c d}\right) N_{s}\left(k-T_{c d}\right)$

(30) $N_{g}(k+1)=N_{0}-N_{s}(k+1)-N_{a}(k+1)-N_{i}(k+1)-N_{c}(k+1)-N_{d}(k+1)$

where $T_{x y z}=T_{x}+T_{y}+T_{z}, N_{s}$ represents the mean number of robots in the search state, $N_{a}$ those in the obstacle avoidance state, $N_{i}$ those in the interference state, $N_{c}$ those in the stickcentering state, $N_{d}$ those in the success dance state, and $N_{g}$ those in the grip state. The $\Delta_{g 2}-$ function can be calculated with Eq. (14) and $\Gamma$-functions with Eq. (15), while the $\Delta_{g 1}$-function is modified as follows:

(31) $\widetilde{\Delta}_{g 1}(k)=p_{g 1}\left[M_{0}-N_{g}(k)-N_{d}(k)\right]$

Equations (25)-(30) can be interpreted in a way similar to that shown for Eqs. (11)-(12). For instance, Eq. (25) indicates that the mean number of robots in search state at any time is decreased by the number of robots that transition to a grip state (grip1 and grip2) and by the number that start avoiding a wall or a teammate; $N_{s}$ is increased by the number of robots that come back from a successful collaboration either as first or second robot (the success dance now has a duration greater than zero), those which come back from an unsuccessful collaboration, and those which finish their wall or robot avoidance maneuver. It is interesting to notice that all states other than search (the default behavior) and grip (calculated with the robots' conservation law) are characterized by a $N_{\mathrm{s}}$ factor (either at the current iteration $k$ or delayed) since they are simple delays like those used in the 2-state system described in Subsection 4.1. However, much like the simplified stick-pulling model described in Subsection 4.2, the coefficients with which the state variable $N_{s}$ is multiplied are time-variable and functions of other state variables or $N_{s}$ itself (see $\Gamma$ - and $\Delta$-functions), thus generating nonlinear coupling between the equations.

As in the case of the 2-state systems described in Section 4, the initial conditions for the DE system are $N(0)=\left[\begin{array}{llllll}N_{0} & 0 & 0 & 0 & 0 & 0\end{array}\right]^{T}$ (all robots are in search state at the beginning of the experiment).

\section{$5.1 \quad$ Results}

We can now compare the results of our microscopic and macroscopic abstraction with those gathered with lower implementation levels, i.e., embodied simulations and real robots. Real robot experiments lasted about 20 minutes (duration of the on-board batteries) and were repeated three times each, while those carried out in the embodied simulator lasted 30 minutes (simulated time) and were repeated ten times. 

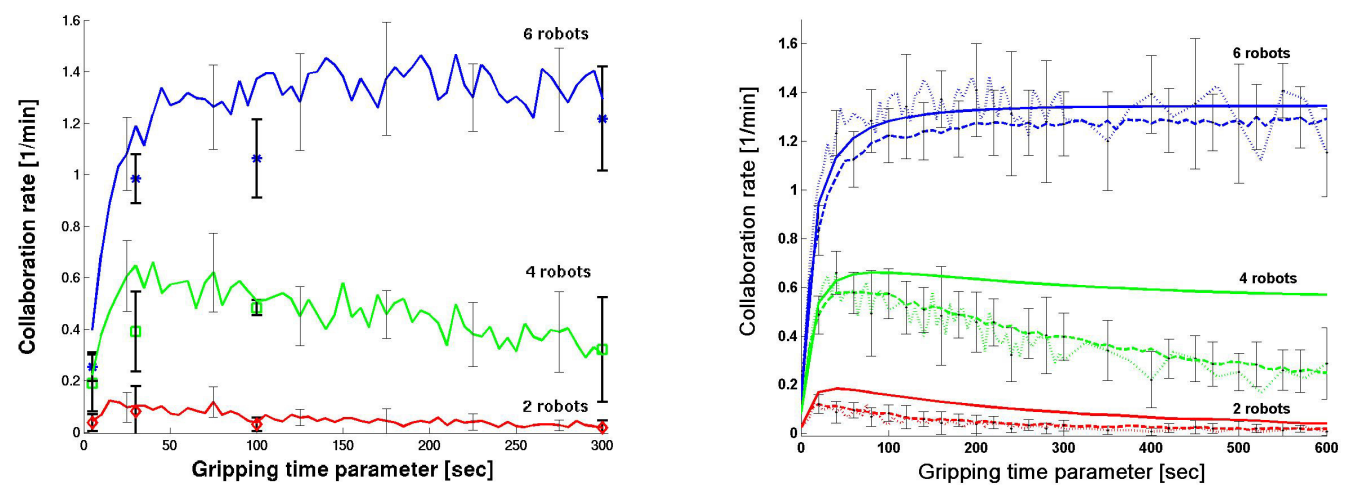

Figure 8: Collaboration rate as a function of the gripping time parameter for group sizes of two, four, and six robots in a $40 \mathrm{~cm}$ radius arena. Left: Results gathered using real robots $\left(\tau_{g}\right.$ $=[5,30,100,300] \mathrm{s})$ and embodied simulations $\left(\tau_{g}=[0: 5: 300] \mathrm{s}\right)$. Right: microscopic and macroscopic models' predictions overlapped with the embodied simulations' results $\left(\tau_{g}=\right.$ [0:5:600] s).

Figure 8, left compares results obtained with real robots and embodied simulations. Although the real robot experiments were repeated only three times, as compared to the ten runs used for embodied simulations, real robots appear to achieve a slightly lower collaboration rate than their corresponding simulated agents. Differences between simulated and real robots' gripper modules are at the origin of this discrepancy. We will discuss this type of problem in detail in Subsection 6.1.

Furthermore, Figure 8, right clearly shows that, in contrast to the macroscopic results,
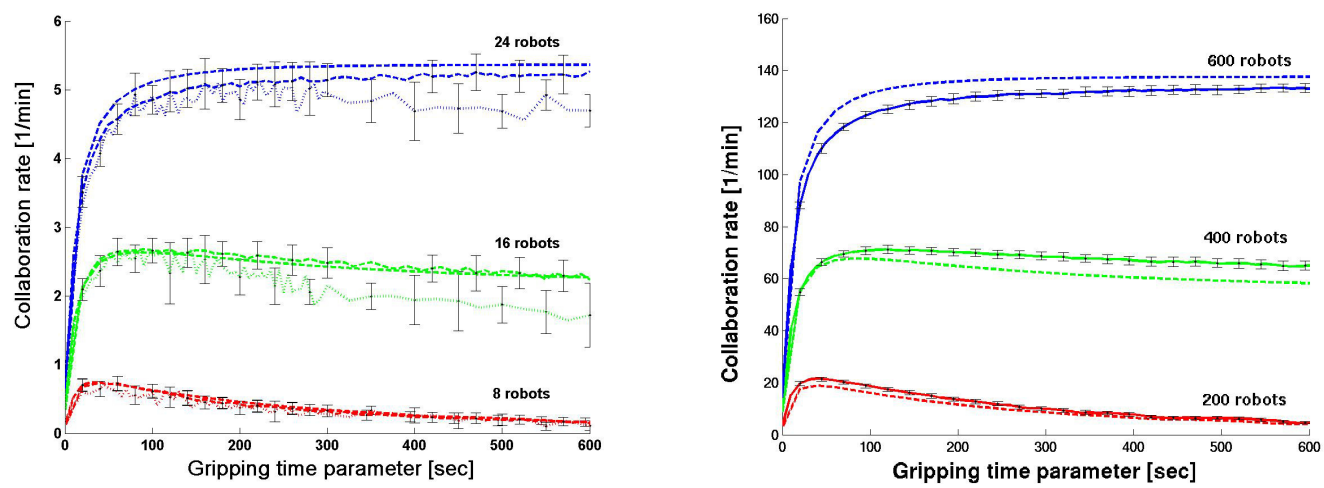

Figure 9 Left: Results of embodied simulations, microscopic and macroscopic models for 8 , 16 , and 24 robots, 16 sticks, and an arena $80 \mathrm{~cm}$ in radius. Right: Predictions obtained using microscopic and macroscopic models for swarms of 200, 400, and 600 robots in an arena $400 \mathrm{~cm}$ in radius.

predictions delivered by the microscopic model are in good quantitative agreement with the data collected using the embodied simulator for all the team sizes.

Much like the results presented in Subsection 4.2.1, these problems are drastically attenuated as soon as we increase the number of robots and sticks, maintaining the same 
density of both items per unit area. Figure 9, left shows a good quantitative agreement among the three simulation levels simply by multiplying the robot and stick quantities by four and increasing the arena size to maintain the same object density, but without changing any implementation details. However, as soon as we multiply the quantities by 100 (see Figure 9, right), we notice that the problem mentioned in Subsection 4.2.1 arises again, but even more accentuated. Section 6 will address again these problems more in detail.

\subsection{Steady-State Analysis}

Like the simplified 2-state system described in Subsection 4.2, the DEs describing the full system are nonlinear and, therefore, we must perform the steady state analysis in the time domain. Using the same method we adopted in Subsection 4.2.2 for Eqs. (25) and (30) and the approximation in time domain introduced in Subsection 4.1.2 for all the delay states of the full system (Eqs. (26)-(29)), we obtain:

(32) $0=-p_{g 1}\left(M_{0}-N_{g}^{*}-N_{d}^{*}\right)+p_{g 1}\left(M_{0}-N_{g}^{*}-N_{d}^{*}\right) \Gamma^{*}+p_{g 2} N_{g}^{*}$

(33) $N_{a}^{*}=T_{a} N_{s}^{*}\left[p_{g 1}\left(M_{0}-N_{g}^{*}-N_{d}^{*}\right) \Gamma^{*}+2 p_{g 2} N_{g}^{*}+p_{w}+p_{R}\right]$

(34) $N_{i}^{*}=T_{i} p_{R} N_{s}^{*}$

(35) $N_{c}^{*}=T_{c} N_{s}^{*}\left[p_{g 1}\left(M_{0}-N_{g}^{*}-N_{d}^{*}\right)+p_{g 2} N_{g}^{*}\right]$

(36) $N_{d}^{*}=T_{d} p_{g 2} N_{s}^{*} N_{g}^{*}$

(37) $N_{g}^{*}=N_{0}-N_{s}^{*}-N_{a}^{*}-N_{i}^{*}-N_{c}^{*}-N_{d}^{*}$

The collaboration rate in steady state becomes:

(38) $C^{*}=p_{g 2} N_{s}^{*} N_{g}^{*}=p_{g 2} N_{s}^{*}\left(N_{0}-N_{s}^{*}-N_{a}^{*}-N_{i}^{*}-N_{c}^{*}-N_{d}^{*}\right)$

Simulating the DE system (25)-(30) long enough until a stationary regime is reached and solving the equation system (32)-(37) are two alternative options for obtaining the full system's steady state vector $N^{*}=\left[\begin{array}{lllllll}N_{s}^{*} & N_{a}^{*} & N_{i}^{*} & N_{c}^{*} & N_{d}^{*} & N_{g}^{*}\end{array}\right]^{\mathrm{T}}$. Both of these operations must be performed numerically. Indeed, if we try to solve the equation system (32)-(37) analytically by substitution and introducing all the results in Eq. (32) we obtain the following transcendental equation:

(39) $P_{0}\left(N_{s}^{*}\right)+P_{1}\left(N_{s}^{*}\right) \Gamma^{*}+P_{2}\left(N_{s}^{*}\right) \Gamma^{* 2}=0$

where $P_{0}, P_{1}$, and $P_{2}$ are second order polynomials in $N_{s}^{*}$ whose coefficients are a function of all the system parameters, i.e., $p_{i j}=p_{i j}\left(M_{0}, N_{0}, p_{g l}, p_{g 2}, p_{w}, p_{R}, T_{a}, T_{i}, T_{c}, T_{d}\right), i$ being the polynomial index $(i=0,1,2)$ and $j$ being the term number in a given polynomial $(j=0,1,2)$.

Like Eq. (18), Eq. (39) can be solved for $\Gamma$ (a quadratic equation here) and therefore also for $T_{g}$. However, unlike the analysis described in Subsection 4.2.2, determining the value of $N_{s}^{*}$, which achieves an optimal collaboration rate, implies we know what values the delay state variables assume in steady state, as shown by Eq. (38). Unfortunately, $N_{a}^{*}, N_{i}^{*}, N_{c}^{*}, N_{d}^{*}$ are, in turn, nonlinearly coupled with $N_{s}^{*}$, preventing us from finding an optimal value without solving (39) for $N_{s}^{*}$. Although we can approximate $\Gamma$ with a McLaurin series 
( $\left.\Gamma^{*} \cong \mathrm{e}^{-T_{g} p_{g 2} N_{s}^{*}}\right)$, Eq. (39) can be solved, to our knowledge, only numerically. This prevents us from formulating analytical expressions for the system bifurcation points (such as that reported in Eq. (24)) or superlinear-linear and linear-sublinear regime transitions (such as those reported in Ijspeert et al. ${ }^{18}$ for the relative collaboration rate, i.e., the collaboration rate normalized over the total number of robots used in the experiment).

\section{Discussion}

In Section 5, we have presented results of the stick-pulling experiment obtained at different levels of implementation: real robots, embodied simulations, microscopic and macroscopic models. In this section, we would like to discuss the problems and subtle effects that arise in moving from one level to a more abstract one. We will conclude this section by discussing the usefulness and limitations of this modeling methodology for optimization purposes.

\subsection{From Real Robots to Embodied Simulations}

Although in general, as demonstrated in several other tasks ${ }^{13,14,27,28}$, the embodied, sensor-based simulator Webots has provided very faithful results, simulation is never reality and several effects due to nonlinear physical laws, noise, small heterogeneities among robots and components are simply neglected in simulation in order to reduce computational cost. In particular, when (collaborative) manipulation is performed with miniature robots like the Khepera, these effects can play a major role: grippers are usually endowed with few degrees of freedom and sensors are affected by a high level of noise.

In the specific case of the stick-pulling experiment, we have observed (but never quantified) that the discrepancies between results obtained using the embodied simulator and those using real robots shown in Figure 8, left have to be attributed to differences in the real gripper module and its corresponding embodied simulation. These differences between simulated and real grippers are two-fold. First, reliability: while the simulated gripper never releases the stick unintentionally (in other words, the trigger for releasing is always either a teammate's help or the internal timeout), the real gripper sometimes drops the stick early due to the noise in measuring the elevation of the arm. Since the presence of a teammate is assessed based on the measurement on the arm elevation sensor ${ }^{\mathrm{h}}$, if this measurement is noisy, the robot performing grip1 may believe that a teammate is helping and release the stick, allowing it to drop into its hole again. The error in elevation is correlated with the arm PI controller and more probable when the arm is in full swing. Although the decision of releasing the stick is based on redundant sensory samples and checks, it still happens from time to time, but does not occur in Webots. Second, body solidity: in Webots 2, collision detection routines are 2D and grippers never get entangled (they actually pass through each other) as they may do in the real world.

Possible fixes for these discrepancies include implementing more realistic sensor noise in the gripper in Webots and using a newer version of the Webots simulator (version 3 or higher), which implements more computationally intensive $3 \mathrm{D}$ collision detection routines and should therefore eliminate the problem of gripper penetration at the price of a slower simulation.

\footnotetext{
$\mathrm{h}$ A systematic positive error on the pre-established position is detected by the first robot when the second robot is pulling the stick as well, see also Subsection 2.3.
} 


\subsection{From Embodied Simulations to Microscopic Models}

A further step of abstraction is that which transforms an embodied, sensor-based agent in a much simpler agent. In the nonspatial, probabilistic, microscopic model presented in this paper, an agent assumes a random position in the arena at each new iteration instead of being characterized by a trajectory. The microscopic agent has a perfectly centered, uniform, and precise range of detection for each object it may encounter in the arena, in contrast to the individual, heterogeneously distributed, noisy sensors available to the real robots and in the embodied simulation. The microscopic agent is characterized by an average speed instead of having a more complex kinematic controller: at lower levels of implementation, accelerations are tightly coupled to sensory readings, whereas the microscopic agent is endowed with a behavior-based controller which can be represented by a precise PFSM whose state-to-state transitions follow either precise durations or are triggered by external events, but never happen with shorter or longer duration due to interrupts or because an event was detected by the sensors earlier or later. In the next three subsections we will discuss the role of some of these important approximations in the accuracy of the predictions delivered by the microscopic model.

\subsubsection{Nonspatial Models}

The modeling methodology assumes a uniform distribution of objects on the arena and no relevance of trajectories or robot spatial distribution to the chosen metric. As long as detection areas do not overlap between the objects placed in the arena (in this case, walls and sticks) and the metric does not specifically address spatiality, this assumption is correct. In order to verify this, we ran several experiments characterized by different stick distributions (see Figure 10) using the embodied simulator. In each of these cases, the predictions of both models were as good as those shown in Figure 8, right, the microscopic model reaching quantitative agreement with the embodied simulations. If needed, the current modeling methodology could be also easily adapted in order to take into account overlapped detection areas. This should be particularly straightforward for non-mobile objects. However, subtle effects due to robot clustering and mutual influence in search and manipulation activities could arise in densely populated scenarios and, as we will show in Subsection 6.2.3, these effects are more difficult to incorporate in the models. Specific robots' distributions (e.g., a specific pattern of movements at the arena's boundary) could also be easily introduced at the price of additional complexity (i.e., more states) in the PFSM(s).
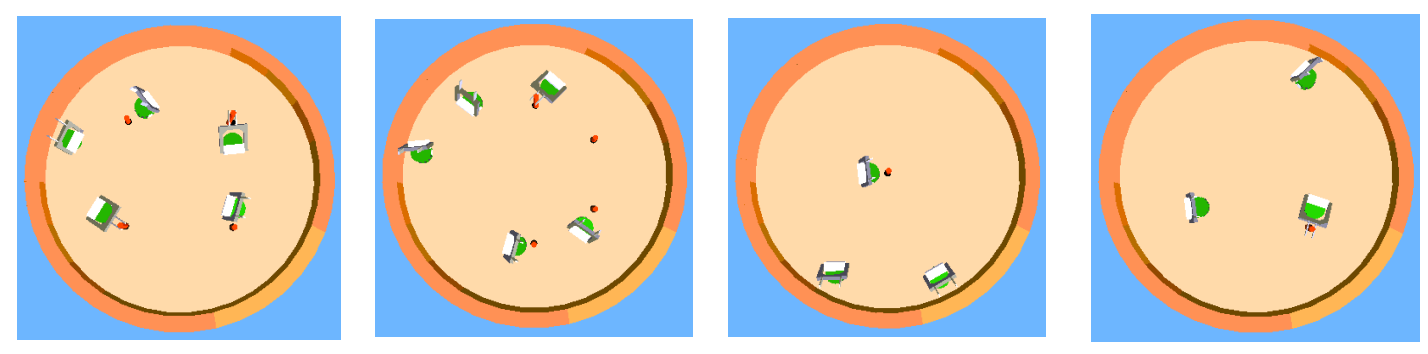

Figure 10: Four examples of implemented stick distributions.

In addition to a robot's position, its orientation may also play a role in the metric considered, particularly when either a specific sensor or actuator has a range comparable to the dimensions of the arena. For instance, in the stick pulling experiment, the introduction of directional communication for attracting robots ${ }^{18,8}$ and vision capabilities in a limited cone of 
view $^{8}$ generates additional difficulties for a nonspatial modeling methodology such as that presented in this paper. Usually, quantitatively correct predictions using nonspatial, probabilistic models can still be achieved, but not without using free parameters ${ }^{18}$.

\subsubsection{Parameter Calibration and Behavioral Granularity}

As seen in Section 3.2, the models' parameters characterize the microscopic robot-torobot and robot-to-environment interactions. They include probabilities of encountering specific objects, sometimes even considering a preferred angle of approach, and delays required by specific maneuvers whose details are uninteresting for the metric considered. This implies, for instance, an effort to summarize belts of noisy, perhaps unevenly-spaced sensors as well as their relative detection and reactive control algorithms with an average detection area or capture an obstacle avoidance maneuver with a mean duration.

In this paper, we used the method adopted in several previous publications, which bases the parameter calibration on simple geometrical considerations and systematic experiments with one or two real robots. Alternative calibration methods have been proposed by other authors. For instance, an interesting one is this proposed by Lerman and Galstyan in a recent paper $^{24}$, a method which also relies on the area swept out by the robots' sensors and normalized by the total arena's area, but without any explicit link between time partitioning and area partitioning based on the smallest object present on the arena. While all these heuristic methods are certainly good attempts in the right direction, none of them has, thus far, taken into account the fact that model parameters, measured with systematic tests at lower levels of implementation, may also be characterized by a measurement error and should, therefore, be introduced in the models as a mean value and corresponding distribution instead of an average value. This is an interesting hypothesis, and one we plan to investigate in the near future, although the complexity of the models and the propagation of errors in nonlinear, time-delayed, systems will not be trivial.

Further difficulties may arise because of the behavioral granularity captured in the microscopic model. The robot controller used in the stick-pulling case study, developed prior to the modeling methodology presented here, can be approximated as a FSM, though certain routines (obstacle avoidance and interference) have been implemented with proximal controllers. Proximal controllers, in our case neural network-based controllers, tightly couple actuators with sensors without passing through a distal representation as, for instance, is the case for behavior-based implementations. Parameters used to describe the states corresponding to such routines (in our case, the duration of obstacle avoidance and interference as well as the probability of detecting an obstacle and a teammate) can still be measured in systematic tests with one or two robots, as mentioned above, even if this implies some inaccuracy. For predicting the collaboration rate, our chosen metric for the stick-pulling task, this approximation is quite sufficient. Consider, however, a simpler system, for example, a controller consisting only of a searching mode and the obstacle avoidance and interference routines used proximal controllers. The description of a state as proposed in this paper would probably no longer be adequate for such controllers; temporal attractors in the state space rather than static state definitions may achieve better results.

\subsubsection{Overcrowded Arenas}

The modeling methodology achieves quantitatively correct predictions of nonspatial metrics based on the assumption that robots are, on average, homogeneously distributed in the arena. As soon as this assumption is no longer valid, such as in an overcrowded scenario, the current methodology reaches its limitations and predictions are no longer quantitatively correct. Another way to explain why an overcrowded scenario breaks the methodology's assumptions is that the models as generated by the methodology are no longer Markovian in this situation. As the arena becomes crowded with robots, though the robots can still move 
around and collaborate, the next transition between controller states is contingent on each robot's trajectory and the trajectories of the surrounding robots. The robots form clusters with overlapping detection areas, which frees space around sticks that can, in turn, be exploited by other robots for successful collaborations. To maintain Markovian assumptions in this situation, we must consider states relative to robot trajectories, which will result in an explosion of complexity of the models, if formulated in the same way we proposed in the methodology presented in this paper.

Figure 11, left illustrates this effect for the microscopic model. The plot shows a clear discrepancy between the microscopic model's and embodied simulation's optimal collaboration rate for group sizes greater than ten robots. While the embodied simulation results reflect this continuing collaboration mitigated by crowding, the microscopic model predicts ever-increasing performance. The continual increase here is due to the fact that the interference area represented by the robots cannot expand into sticks' and wall's detection areas and will therefore saturate to a maximal value corresponding to the free space available in the arena (see Eq. (1)). An alternative option, which allows the robots' detection area to grow until the calculated probability of encountering another robot is one, will also fail to deliver correct predictions since, at a certain point, the collaboration rate disappears ${ }^{18,31}$ (in the model, the robots can only execute interference maneuvering) while in the embodied simulation performance continues to be greater than zero, as shown in Figure 11, left.

Figure 11, right shows the problem from another perspective. The piecewise linear approximation we do in the model for the probability of encountering another robot per time step is also approximately correct for teams of up to ten robots, but then, in the saturation
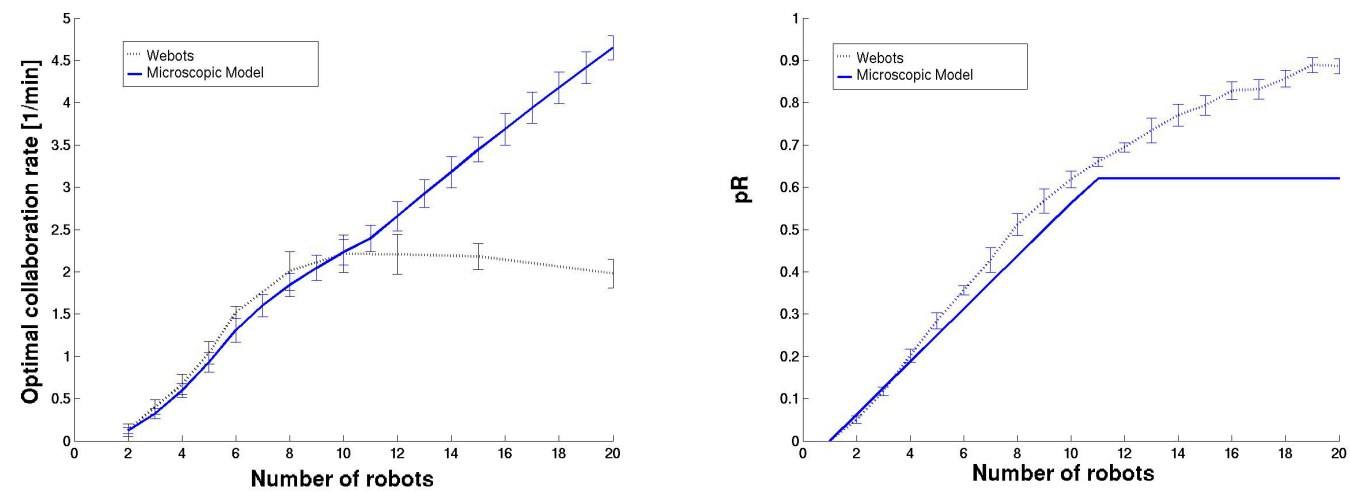

Figure 11: Left: Comparison of the prediction obtained using embodied simulations and the microscopic model in an overcrowded arena (up to 20 robots in an arena of $40 \mathrm{~cm}$ in radius). For each group size, the collaboration rate achieved after optimization of $\tau_{g}$ (systematic search) is plotted. Right: Probability of encountering a robot in the embodied simulator as compared to the linear approximation used in the modeling methodology.

phase, the model's curve diverges from the likelihood measured using embodied simulations.

\subsection{From Microscopic to Macroscopic Models}

As seen in Subsections 4.2.1 and 5.1, the microscopic and macroscopic models of a distributed manipulation experiment, although they rely on the same abstraction of the individual agent, may deliver slightly divergent predictions. The discrepancies are 
fundamentally due to the fact that typical models of a distributed manipulation experiment are constituted by nonlinear, often time-delayed DEs and that the average quantities predicted by the macroscopic models cannot simply be calculated from the linear combination of the individual PFSMs constituting the microscopic model. This already affects the predicted mean value of state variables, which in turn introduces more or less relevant discrepancies in the metric used for evaluating the swarm performance. For instance, the discrepancies between microscopic and macroscopic models in the collaboration rate (based on $N_{s}$ and $N_{g}$ state variables) appear to be slightly more important in the full-system model (Figure 8 and Figure 9) than in the simplified one (Figure 6, left column) because in the simplified model, inaccuracies in the $N_{s}$ state variable can be directly compensated with opposite sign in the $N_{g}$ state variable, while this is only partially possible in the full-system model.

Further discrepancies may have a combinatorial origin: the microscopic model correctly

calculates the allocation of robots at limited, shared manipulation sites by keeping track of each modification of the environment generated by the actions of the robots. The macroscopic model instead summarizes the states of the robotic swarm and environment with expected value quantities and closed form expressions, a representation which is often only an approximation of combinatorial series.

Unfortunately, it is at this point difficult to draw general quantitative conclusions on discrepancies between microscopic and macroscopic models. Only after having carefully analyzed how errors propagate as a function of the nonlinearities characterizing a given system, we will be able to evaluate more precisely the effects of this further level of abstraction. Moreover, it is currently unclear whether or not we will be able to identify a set of nonlinearities systematically arising in distributed manipulation or, even more generally, swarm robotic experiments for which we could develop ad hoc but accurate error estimation procedures.

\subsection{Modeling as Tool for Optimization}

The modeling methodology presented in this paper can be useful also from optimization point of view. First, the models presented here deliver results in time lapses that are at least four orders of magnitude shorter than a corresponding embodied simulation. Second, abstraction in general allows researchers to understand the role of key system parameters, generalize and analyze underlying principles, and sometimes even enables mathematical tractability and resituates intuitive considerations, as we have shown in Subsections 4.1.2 and 4.2.2. However, in swarm systems, which base their principles of self-organized, collective behavior on multiple interactions among individuals and between individuals and environment, low-level system parameters such as the sensory configuration, body shape, proximal control parameters characterizing reactive behavior, and individuals' heading and positions may also play an important role, which we may neglect if we try to optimize the system at higher level. In other words, searching the optimal solution in a larger parameter space, including also low-level hardware and software parameters, may achieve better results than those explored with a more or less abstract model. Two concrete examples may illustrate this point.

A first example is concerned with collective plume tracing ${ }^{14}$. In this experiment, due to the highly stochastic nature of robot-plume interactions, the unavailability of suitable plume models for our specific task conditions, and the relevance of robots' heading and positions, we have thus far only been able to create models of the whole system characterized by several free parameters (see Hayes ${ }^{15}$ for details), although all of them having a clear intuitive meaning. This has prevented us not only to achieve, in the best case, only a qualitative system optimization but also to directly encode all the key controller parameters in the models. Nevertheless, we were remarkably successful in system optimization with an ad hoc 
reinforcement learning algorithm combined with embodied simulations ${ }^{16}$ since no explicit and reliable model of the system is required by a machine-learning method.

A second example comes from additional experiments we performed in the framework of the stick-pulling experiment. As Ijspeert et al. ${ }^{18}$ have shown using systematic search and embodied simulations and as $\mathrm{Li}$ et al. ${ }^{25}$ have shown using a simple learning algorithm combined with the same microscopic model we presented in this paper, a homogeneous swarm may not necessarily achieve the best performance. Sub-swarms of specialists in grip1 and grip2 may, for instance, outperform an optimized homogeneous swarm, depending on the sticks-to-robots ratio. Generally speaking, at the macroscopic level, either we know the number of castes in advance and we introduce a new set of DEs for each new type of agent involved in the system, or it would be impossible to explore heterogeneous solutions. Therefore, to explore heterogeneous solutions, microscopic models combined with machinelearning algorithms appear to be a more efficient solution than macroscopic models preestablishing the heterogeneity of the system (often, considering exclusively the homogeneous case).

Finally, as shown in Subsection 5.2, even if we are able to produce quantitatively correct analytical models, nonlinearities and complexity of a real system, even a simple one as that used in the stick-pulling experiment, often prevent us from going further in the analysis (and indirectly in the optimization process) with the mathematical tools currently available.

\section{Conclusion and Outlook}

In this paper we presented a methodology for generating nonspatial, probabilistic microscopic and macroscopic models of swarm robotic systems. The methodology was explicitly designed for distributed manipulation experiments and we therefore supported the discussion with a case study - the stick-pulling experiment - belonging to this class. We have shown that models can deliver not only quantitatively correct predictions in periods at least four order of magnitude shorter than other popular simulation tools, such as sensor-based, embodied simulation, but can also allow for better understanding of the system properties and, in some cases, even enable mathematical analysis of the system.

We have also discussed several difficulties one may encounter in moving from one level of implementation (real robots, embodied simulations, microscopic and macroscopic models) to a more abstract one. It is worth noticing that all these abstraction steps were hand-coded, exploiting a combination of engineering, heuristic, and systematic tests. Although in this paper we did not compare the results of models with those obtained at lower-level implementations using non-parametric statistical tests (something we did in previous publications ${ }^{29,27}$ ), we do not believe that we will gain further insight into how to resolve the difficulties in moving from one level of implementation to another in this way. However, statistical tests combined with algorithms that can systematically explore possible abstraction options and, at the same time, verify the statistical impact of each of them on a chosen metric may allow us to achieve fundamental breakthroughs in the modeling methodology. This approach would be also well-suited when models' parameters are described not only as a mean value but rather as a distribution characterized by central tendencies (e.g., mean and standard deviation for a Gaussian distribution). First attempts in this direction for different purposes have been proposed by Goldberg and Mataric ${ }^{11}$ (automatic generation of behavioral FSM) and Zhang et al. ${ }^{42}$ (generation of Probability Density Functions for automatic design of sensory configurations).

Further challenges are concerned with overcoming current limitations of and extending the current modeling methodology to other classes of experiments and systems. Boundary conditions such as overcrowded scenarios or specific environments inducing heterogeneous robots' distributions, non-behavior-based controllers (e.g., proximal, neural controllers), more 
sophisticated individual capabilities (e.g., learning, peer-to-peer wireless communication, and navigation capabilities), distributed sensing tasks (e.g., mapping, searching), and more complex systems (e.g., mixed insect-robot societies ${ }^{43}$ ) are examples of problems we intend to address using enhanced probabilistic modeling methodologies in the next few years.

\section{Acknowledgements}

The authors wish to thank Ling Li for helpful discussions on this work and for having suggested the correction with the factor " $-\mathrm{N}_{\mathrm{d}}$ " in the full-system model. The authors wish also to thank Kristina Lerman and Aram Galstyan for having stimulated the discussion and fundamentally contributed to macroscopic modeling in the multi-agent systems.

This work was carried out when all the three authors were part of the Collective Robotics Research Group at the California Institute of Technology (see also http://swis.epfl.ch). It was supported primarily by the Engineering Research Centers Program of the American National Science Foundation under Award Number EEC-9402726 and in part by the TRW Foundation. AM is currently sponsored by a Swiss National Science Foundation professorship.

\section{References}

1. Agassounon, W., Martinoli, A. and Goodman, R. M., "A Scalable, Distributed Algorithm for Allocating Workers in Embedded Systems". Proc. of the IEEE Conf. on System, Man and Cybernetics SMC-01, October 2001, Tucson, AR, USA, pp. 3367-3373.

2. Beckers R., Holland O. E., and Deneubourg J.-L., "From Local Actions to Global Tasks: Stigmergy and Collective Robotics". In Brooks R. and Maes P., editors, Proc. of the Fourth Workshop on Artificial Life, Boston, MA, July, 1994, The MIT Press, pp. 181-189.

3. Beni G. and Wang J., "Swarm Intelligence". Proc. of the Seventh Annual Meeting of the Robotics Society of Japan, Tokyo, Japan, 1989, pp. 425-428.

4. Billard A., Ijspeert A. J., and Martinoli A., "A Multi-Robot System for Adaptive Exploration of a Fast Changing Environment: Probabilistic Modelling and Experimental Study". Special Issue on Adaptive Robots, Torras C., editor, Connection Science, Vol. 11, No. 3/4, pp. 359-379, 1999.

5. Bonabeau E., Dorigo M., and Theraulaz G., "Swarm Intelligence: From Natural to Artificial Systems". SFI Studies in the Science of Complexity, Oxford University Press, New York, NY, 1999.

6. Chauvin R. and Janin P., "Facteurs de direction et d'excitation au cours de l'accomplissement d'une tâche chez Formica polyctena". Insectes Sociaux, Vol. 22, pp. 199-206, 1975.

7. Das A. K., Fierro R., Kumar V., Ostrowoski J. P., Spletzer J., and Taylor C. J., “A Vision-Based Formation Control Framework". Special Issue on Advances in Multi-Robot Systems, Arai T., Pagello E., and Parker L. E., editors, IEEE Trans. on Robotics and Automation, Vol. 18, No. 5, pp. 813-825, 2002.

8. Easton K. and Martinoli A., "Efficiency and Optimization of Explicit and Implicit Communication Schemes in Collaborative Robotics Experiments". Proc. of the 2002 IEEE Int. Conf. on Intelligent Robots and Systems IROS-02, September-October 2002, Lausanne, Switzerland, pp. 2795-2800.

9. Fredslund J. and Matarić M. J., "General Algorithm for Robot Formations Using Local Sensing and Minimal Communication". Special Issue on Advances in Multi-Robot Systems, Arai T., Pagello E., and Parker L. E., editors, IEEE Trans. on Robotics and Automation, Vol. 18, No. 5, pp. 837-846, 2002.

10. Gerkey B. P. and Matarić M. J., "Sold!: Auction Methods for Multirobot Coordination". Special Issue on Advances in Multi-Robot Systems, Arai T., Pagello E., and Parker L. E., editors, IEEE Trans. on Robotics and Automation, Vol. 18, No. 5, pp. 758-768, 2002.

11. Goldberg D. and Matarić M. J., "Mobile Robot Group Coordination Using a Model of Interaction Dynamics". Proc. of the SPIE: Sensor Fusion and Decentralized Control in Robotic Systems II, 1999, Boston, MA, pp. 63-73.

12. Grassé P.-P., "La reconstruction du nid et les coordinations inter-individuelles chez Bellicositermes Natalensis et Cubitermes sp. La théorie de la Stigmergie: essai d'interprétation du comportement des termites constructeurs". Insectes Sociaux, Vol. 6, pp. 41-80, 1959.

13. Hayes A. T., Martinoli A., and Goodman R. M., "Comparing Distributed Exploration Strategies with Simulated and Real Autonomous Robots". In L. E. Parker, G. Bekey, and J. Bahren, editors, Proc. of the Fifth Int. Symp. on Distributed Autonomous Robotic Systems DARS-00, October, 2000, Knoxville, TN, pp. 261-270. 
14. Hayes A. T., Martinoli A., and Goodman R. M., "Distributed Odor Source Localization". Special Issue on Artificial Olfaction, Nagle H. T., Gardner J. W., and Persaud K., editors, IEEE Sensors, Vol. 2, No. 3, pp. 260-271, 2002.

15. Hayes A. T., "Self-Organized Robotic System Design and Autonomous Odor Localization". Unpublished doctoral manuscript, Caltech, CA, June, 2002. See also http://www.coro.caltech.edu/People/athayes/athayes.html.

16. Hayes A. T., Martinoli A., and Goodman R. M., "Swarm Robotic Odor Localization: Off-Line Optimization and Validation with Real Robots". Special issue on Biological Robotics, McFarland D., editor, Robotica, Vol. 21, Issue 4, pp. 427-441, 2003.

17. Holland O.E. and Melhuish C., "Stigmergy, Self-Organisation, and Sorting in Collective Robotics". Artificial Life, Vol. 5, pp.173-202, 1999.

18. Ijspeert A. J., Martinoli A., Billard A., and Gambardella L.M., "Collaboration through the Exploitation of Local Interactions in Autonomous Collective Robotics: The Stick Pulling Experiment”. Autonomous Robots, Vol. 11, No. 2, pp. 149-171, 2001.

19. Kazadi S., Abdul-Khaliq A., and Goodman R. M, "On the Convergence of Puck Clustering Systems". Robotics and Autonomous Systems, Vol. 38, No. 2, pp. 93-117, 2002.

20. Kelly I. D., Keating D. A., "Flocking by the Fusion of Sonar and Active Infrared Sensors on Physical Autonomous Mobile Robots". Proc. of the Third Conf. on Mechatronics and Machine Vision in Practice, Gaimardes, Portugal, 1996, Vol. 1, pp. 1-4.

21. Krieger M. J. B. and Billeter J.-B., "The Call of Duty: Self-Organised Task Allocation in a Population of up to Twelve Mobile Robots". Robotics and Autonomous Systems, Vol. 30, No. 1-2, pp. 65-84, 2000.

22. Kube C. R. and Bonabeau E., "Cooperative Transport by Ants and Robots". Robotics and Autonomous Systems, Vol. 30, No. 1-2, pp. 85-101, 2000.

23. Lerman K., Galstyan A., Martinoli A., and Ijspeert A. J., "A Macroscopic Analytical Model of Collaboration in Distributed Robotic Systems". Artificial Life, Vol. 7, No. 4, pp. 375-393, 2001.

24. Lerman K. and Galstyan A., "Mathematical Model of Foraging in a Group of Robots: Effect of Interference". Autonomous Robots, Vol. 13, pp. 127-141, 2002.

25. Li L., Martinoli A., and Abu-Mostafa Y., "Emergent Specialization in Swarm Systems". In H. Yin, N. Allinson, R. Freeman, J. Keane, and S. Hubbard, editors, Proc. of the Third Int. Conf. on Intelligent Data Engineering and Automated Learning, August 2002, Manchester, UK. Lecture Notes in Computer Science (2002), Vol. 2412, pp. 261-266.

26. Martinoli A. and Mondada F., "Collective and Cooperative Group Behaviours: Biologically Inspired Experiments in Robotics". In Khatib O. and Salisbury J. K., editors, Proc. of the Fourth Int. Symp. on Experimental Robotics, Stanford, June, 1995, Lecture Notes in Control and Information Sciences, pp. 3-10.

27. Martinoli A., Ijspeert A. J., and Mondada F., "Understanding Collective Aggregation Mechanisms: From Probabilistic Modelling to Experiments with Real Robots". Robotics and Autonomous Systems, Vol. 29, pp. 51-63, 1999.

28. Martinoli A., Ijspeert A. J., and Gambardella L. M., "A Probabilistic Model for Understanding and Comparing Collective Aggregation Mechanisms”. In Floreano D., Mondada F., and Nicoud J.-D., editors, Proc. of the Fifth European Conf. on Artificial Life, September, 1999, Lausanne, Switzerland, Lectures Notes in Computer Science, pp. 575-584.

29. Martinoli A., "Swarm Intelligence in Autonomous Collective Robotics: From Tools to the Analysis and Synthesis of Distributed Control Strategies". Unpublished doctoral manuscript, EPFL Ph.D. Thesis Nr. 2069, October 1999, Lausanne, Switzerland. Downloadable at: http://www.coro/caltech/edu/people/alcherio/am_pub.

30. Martinoli A., Theraulaz G., and Deneubourg J.-L., "Quand les robots imitent la nature". La Recherche, No. 358, pp. 56-62, Novembre, 2002.

31. Martinoli A. and Easton K., "Modeling Swarm Robotic Systems". In Siciliano B. and Dario P., editors, Proc. of Eighth Int. Symp. on Experimental Robotics, July 2002, Sant'Angelo d'Ischia, Italy. Springer Tracts in Advanced Robotics (2003), pp. 297-306.

32. Martinoli A. and Easton K. "Optimization of Swarm Robotic Systems via Macroscopic Models". In Schultz A. C., Parker L. E., and Schneider F. E., editors, Proc. of the Second Int. Workshop on Multi-Robots Systems, March, 2003, Washington, DC, U.S.A., pp. 181-192.

33. Matarić M. J., "Interaction and Intelligent Behavior". Unpublished doctoral manuscript, Dept. of Electrical Engineering and Computer Science, MIT, Cambridge, MA, 1994.

34. Michel O., "Webots: Symbiosis Between Virtual and Real Mobile Robots". In Heuding J.-C., editor, Proc. of the First Int. Conf. on Virtual Worlds, Paris, France, July, 1998, Springer Verlag, pp. 254-263. See also http://www.cyberbotics.com/webots/.

35. Mondada F., Franzi E., and Ienne P., "Mobile Robot Miniaturization: A Tool for Investigation in Control Algorithms". In Yoshikawa T. and Miyazaki F., editors, Proc. of the Third Int. Symp. on Experimental 
Robotics, Kyoto, Japan, October, 1993, Lecture Notes in Control and Information Sciences, Springer Verlag, pp. 501-513.

36. Parker L. E., "Lifelong Adaptation in Heterogeneous Multi-Robot Teams: Response to Continual Variation in Individual Robot Performance". Autonomous Robots, Vol. 8, pp. 239-267, 2000.

37. Parrish J. K. and Hamner W. M., “Animal Groups in Three Dimensions". Cambridge University Press, 1997.

38. Sugawara K. and Sano M., "Cooperative Accelleration of Task Performance: Foraging Behavior of Interacting Multi-Robots System”. Physica D, Vol. 100, pp. 343-354, 1997.

39. Sugawara K., Sano M., Yoshihara I., and Abe K., "Cooperative Behavior of Interacting Robots". Artificial Life and Robotics, No. 2, pp. 62-67, 1998.

40. Weigel T., Gutmann J.-S., Dietl M., Kleiner A., and Nebel B., "CS Freiburg: Coordinating Robots for Successful Soccer Playing”. Special Issue on Advances in Multi-Robot Systems, Arai T., Pagello E., and Parker L. E., editors, IEEE Trans. on Robotics and Automation, Vol. 18, No. 5, pp. 685-699, 2002.

41. Yamauchi B., "Decentralized Coordination for Multi-robot Exploration". Robotics and Autonomous Systems, Vol. 29, No. 1, pp. 111-118, 1999.

42. Zhang Y., Martinoli A., and Antonsson E. K., "Evolutionary Design of a Collective Sensory System”. In H. Lipson, E. K. Antonsson, and J. R. Koza, editors, Proc. of the 2003 AAAI Spring Symposium Series on Computational Synthesis, Stanford, CA, March 2003, pp. 283-290.

43. "Leurre", European Commission Project IST-2001: http://leurre.ulb.ac.be/.

\section{Index to Multi-Media Extensions}

The multi-media extension to this article can be found online by following the hyperlinks from www.ijrr.org.

\begin{tabular}{|l|l|l|}
\hline Extension & $\begin{array}{l}\text { Media } \\
\text { Type }\end{array}$ & Description \\
\hline 1 & Video & $\begin{array}{l}\text { Short movie of the stick pulling task performed by six real robots in } \\
\text { an arena } 40 \mathrm{~cm} \text { in radius endowed with four sticks }\end{array}$ \\
\hline
\end{tabular}

